\title{
Multi-Objective Optimization of Semi-submersible Platforms Using Particle Swam Optimization Algorithm Based on Surrogate Models
}

\author{
Wenzhen Qiu ${ }^{\mathrm{a}, \mathrm{b}}$, Xingyu Song ${ }^{\mathrm{a}, \mathrm{b}}$, Kaiyuan Shi ${ }^{\mathrm{a}, \mathrm{b}}$, Xinshu Zhang ${ }^{\mathrm{a}, \mathrm{b}, *}$, Zhiming Yuan ${ }^{\mathrm{c}}$, Yunxiang \\ You $^{\mathrm{a}, \mathrm{b}}$ \\ ${ }^{a}$ State Key Laboratory of Ocean Engineering, Shanghai Jiao Tong University, \\ Shanghai, 200240, China \\ ${ }^{b}$ Collaborative Innovation Center for Advanced Ship and Deep-Sea Exploration (CISSE), \\ Shanghai, 200240, China \\ ${ }^{c}$ Department of Naval Architecture, Ocean and Marine Engineering, University of Strathclyde, \\ Glasgow, G4 OLZ, UK
}

\begin{abstract}
An Innovative Semi-submersible platform Optimization Program (ISOP) has been developed to solve the multiobjective optimization problem for semi-submersible platforms (SEMI). Three types of SEMIs, including semisubmersible floating production unit (SEMI FPU), heave and vortex induced motion (VIM) suppressed semisubmersible (HVS) and semi-submersible floating drilling unit (SEMI FDU) are selected for case studies. The hydrodynamic performances of three types of semi-submersible platforms are analyzed by using panel method and Morison's equation. In order to improve the computing efficiency, the hydrodynamic performances for different hull forms during optimization process are estimated by the surrogate models, which are built by artificial neural network prediction method and Inverse Multi-Quadric (IMQ) radial basis function (RBF). The accuracy of surrogate models is ensured by performing leave-one-out cross validation (LOOCV). The most probable maximum (MPM) heave motion and total weight, representing the safety and economy, respectively, are chosen as the two objectives for optimization. The transverse metacentric height, the MPM surge motion, and the most probable minimum (MPMin) airgap are selected as constraints. Based on surrogate models, multi-objective particle swarm optimization (MOPSO) is employed to search for the Pareto-optimal solutions. A Computational Fluid Dynamics (CFD) tool is adopted to validate the proposed model for the prediction of the motion responses. By comparing the obtained Pareto-optimal solutions with the initial design using simple panel method plus Morison's equation, it is confirmed that the MPM heave motions for SEMI FPU, HVS and SEMI FDU can be suppressed by up to $12.68 \%, 11.92 \%$, and $14.96 \%$, respectively, and the total weights can be reduced by up to $12.16 \%, 13.00 \%$, and $24.91 \%$, respectively. Through the detailed analyses of optimization results, the most efficient design strategies for semi-submersible platforms are discussed and proposed.
\end{abstract}

\section{Introduction}

Over the past decades, floating production platforms, including Floating Production Storage and Offloading (FPSO), Semi-submersible platform (SEMI), Tension Leg Platform (TLP) and Spar, are widely applied for marine engineering (API RP 2SK, 2005). Comparing to the other concepts, SEMI not only has the advantage

*Corresponding author; Director, Innovative Marine Hydrodynamics Laboratory (iMHL), Shanghai Jiao Tong University.

Email address: xinshuz@sjtu.edu.cn (Xinshu Zhang) 
of large deck space, but also can adapt to a wide range of water depth. Moreover, SEMI can be fabricated in a number of shipyards worldwide and be integrated with the topside in a yard instead of performing installation and integration offshore. Therefore, SEMI becomes increasingly popular for oil/gas exploration and offshore wind energy extraction. However, in order to achieve better global motion performance and reduce the fabrication cost, it is critical to optimize the hull form.

The traditional design process of platform can usually be categorized into three phases: conceptual design, basic design and detailed design. During the conceptual design phase, the form of columns and pontoons, as well as the arrangement of wellhead and production riser are usually determined based on the functional requirements of the platform. Once the conceptual design is completed, the general layout of the platform can be determined, which will be followed by basic design with more detailed analyses to seek the best hydrodynamic performances with the most economical solution. In engineering practice, this type of hull sizing is an iterative process, which is so-called 'design spiral' (API RP 2T, 1997).

In order to search for offshore floating structures with excellent hydrodynamic performances, many researches have been carried out on the optimization for platform. Akagi et al. (1984) developed a computer-aided design method to seek the optimal semi-submersible hull form, in which generalized reduced gradient algorithm (GRGA) was employed. In the study, the geometrical shape of semi-submersible was simplified as several cylinders, and the potential flow theory was applied to evaluate the hydrodynamic performances. Nishimoto and Leite (1993) investigated the effects of 'Dog-bone' concept and blisters on semi-submersible for the reduction of heave motion. Clauss and Birk (1994) and Lee et al. (2007) confirmed that the lateral keels on pontoons and the blisters on columns for SEMI can reduce the heave motion. Clauss and Birk (1996) adopted nonlinear programming approach (NLP) to seek the optimal solutions of three different types of platforms, including gravity base structure (GBS), TLP, and SEMI. Vannucci (1996) simplified the structure of a traditional TLP platform into a single-column platform to reduce the design variables. A square heave plate was added to the bottom of the single cylinder as equivalent damping of the floating tank, while a square plate with weight was added to the top of the single cylinder for equivalent mass of superstructures. The optimal TLP with minimum weight was obtained, which satisfied the stability and motion constraints. Cermelli et al. (2004) proposed to use heave plates to reduce the wave-induced heave response for a minimal floating offshore platform.

$\mathrm{Xu}$ (2011) proposed a HVS semi-submersible concept, which redistributes displacement in pontoons and the base of columns, so that both the heave motion and VIM were reduced. Williams (2012) took heave motion as the objective function to optimize the hull and the mooring system in deep water. The stability of the towing, installation and operation, and the airgap in the extreme survival condition were considered as the constraints. Park et al. (2015) took heave motion response and total steel weight as objectives to optimize a SEMI FPU. As constraints, the transverse metacentric height was larger than $8 \mathrm{~m}$, and the minimum airgap was set to $2 \mathrm{~m}$. The final Pareto solutions were obtained by assigning weights for different objectives. Kim and Jang (2016) adopted Simulated Annealing (SA) to perform multi-objective optimization on a TLP. The two objectives were heave motion and weight of the platform. The constraints were the maximum surge, airgap, the minimum tension at bottom part and the maximum fatigue damage. The maximum fatigue damage for 25 years was less than 0.1. Sugita and Suzuki (2016) adopted Genetic Algorithm (GA) and Simulated Annealing (SA) algorithm to 
optimize a TLP. In the study, the objective functions were hull weight and tendon weight, and the constraints were the transverse metacentric height and airgap. Zhang et al. (2017) performed an extensive parametric study for a typical SEMI FPU, providing a benchmark for the future automated hull form optimization. They found that when the draft of platform increases, the MPM heave motion reduces. However, when the column width, pontoon width, or pontoon height gradually decreases, the MPM heave motion also reduces. More recently, Zhang et al. (2018) applied Non-dominated Sorting Genetic Algorithm II (NSGAII) to find the Pareto-optimal solutions for a TLP. In that study, hull draft, column spacing, column diameter, and pontoon size were selected as design variables with the maximum dynamic tension and total weight being the two objectives.

The principal focus of this paper is to adopt MOPSO to search for the optimal hull configurations for three types of SEMIs to achieve better global motion performances and economy. In order to improve the computing efficiency, the surrogate models are employed to estimate the hydrodynamic performances for different hull forms, instead of the direct numerical simulations. Pareto-optimal solutions have been achieved by using MOPSO, instead of converting to a single-objective optimization problem by using weighted functions. These computational results have been validated by comparing against the direct numerical simulations. The hull sizing strategy will be discussed based on the optimization results.

\section{Mathematical formulation}

In the present study, three-dimensional potential flow theory is adopted to compute the wave loads acting on large-scale structural components. Morison's equation is applied to compute the viscous drag force. Based on the design of experiment (DOE) theories, a set of sample points with different design variables can be assigned (Anderson and Whitcomb, 1970). Through the model generation and mass estimation modules, the transverse metacentric height, hydrodynamic performances, airgap and total structural weight of the platform have been obtained. Surrogate models can be built based on the obtained results of the sample points. In order to ensure the accuracy of predictions, the leave-one-out cross validation (LOOCV) is employed to find formal parameters for radial basis function (RBF). These surrogate models and MOPSO are combined to search for the optimization solutions rapidly. The heave motion and total structural weight are selected as objective functions with three constraints including surge motion, airgap and transverse metacentric height. The transverse metacentric height is equal to the longitudinal metacentric height for SEMI FPU and HVS semi-submersible, while the transverse metacentric height is less than the longitudinal metacentric height for SEMI FDU. Therefore, the transverse metacentric height is selected as constraint in the present study. Finally, Pareto-optimal solutions have been obtained and validated by comparing to the direct numerical simulations. The flowchart of the overall process is illustrated in Figure 1.

\subsection{Hydrodynamic computational model}

\subsubsection{Panel method}

In frame of potential flow theory, it is assumed that the fluid is inviscid and incompressible, and the fluid motion is irrotational, so that panel method can be adopted to compute the wave loads. It is assumed that the amplitudes of wave and motion of body are small compared with the cross section of body. The fluid field and coordination systems are illustrated in Figure 2. 


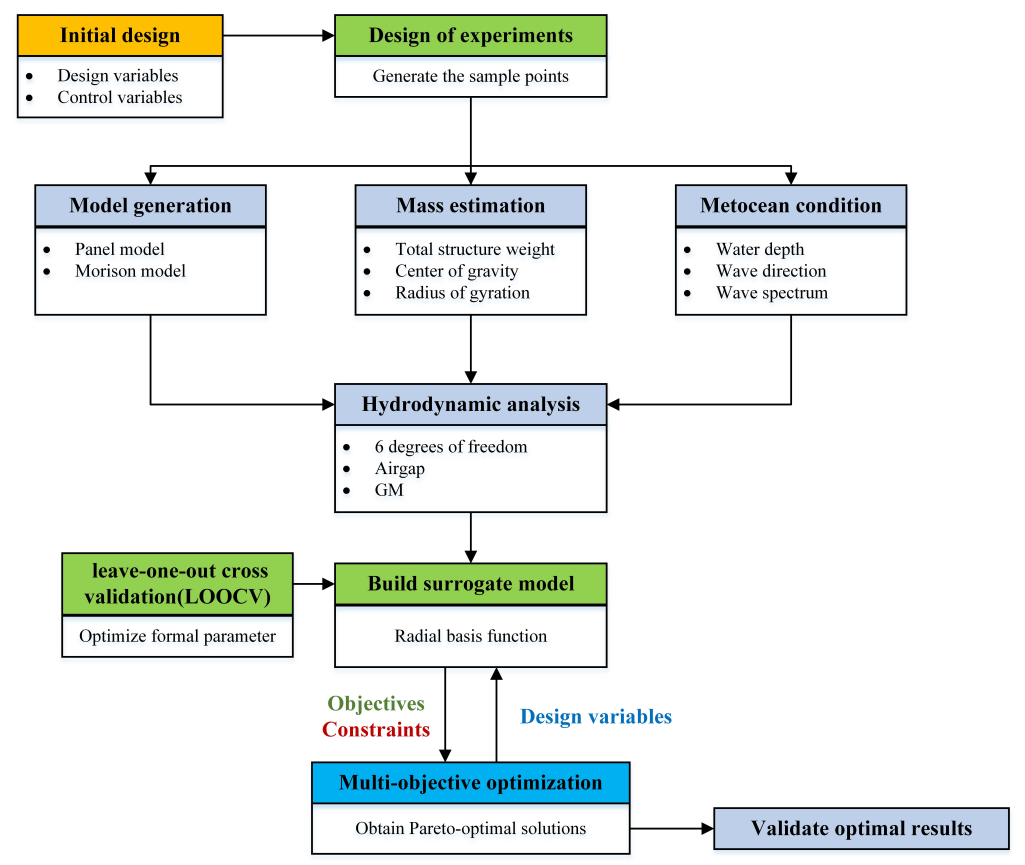

Figure 1: Flowchart of overall optimization process. Yellow module: an initial design obtained from conceptual design phase; Green modules: construction of surrogate models; Gray modules: numerical simulations for hydrodynamic estimation; Blue modules: iterative optimization process.

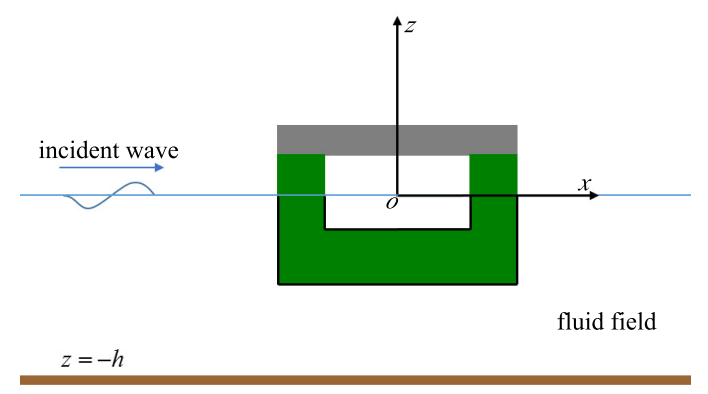

Figure 2: The fluid field and coordination systems. (Two coordinate systems are employed: the $\boldsymbol{x}$ system is fixed to the mean position of the floating body, and the $\boldsymbol{x}_{\mathbf{0}}$ system is fixed to the moving hull. The x-axis points to the direction of platform east and the z-axis points upward. The origin of the coordinate system is located at the intersection of the symmetry axis of the platform and the calm water surface.)

The velocity potential $\phi$ should satisfy Laplace equation. In addition, boundary conditions including linearized free-surface boundary condition, seabed boundary condition, body boundary condition and far-field radiation condition should be satisfied.

Governing equation,

$$
\nabla^{2} \phi=\frac{\partial^{2} \phi}{\partial x^{2}}+\frac{\partial^{2} \phi}{\partial y^{2}}+\frac{\partial^{2} \phi}{\partial z^{2}}=0
$$

On the calm water surface $(z=0)$,

$$
-\omega^{2} \phi+g \frac{\partial \phi}{\partial z}=0
$$

On the seabed $(z=-h)$,

$$
\frac{\partial \phi}{\partial z}=0
$$


On the wetted body surface,

$$
\frac{\partial \phi}{\partial n}=\mathbf{V} \cdot \mathbf{n}
$$

On far-field boundary,

$$
\lim _{r \rightarrow \infty} \sqrt{r}\left(\frac{\partial \phi}{\partial r}-i k \phi\right)=0
$$

where $\mathbf{V}$ is the hull velocity; $\mathbf{n}$ is the normal vector of the hull surface; $r$ is the distance to the center of the hull; $k$ is the wavenumber; $\omega$ is the wave frequency; $g$ is gravity acceleration. The mean wetted surface of the platform is discretized into a number of flat panels, assuming that the source strength on each panel is constant. The governing equation and boundary conditions are employed to solve the source strengths of all the panels. Based on the obtained source strengths, the velocity potentials and hydrodynamic pressure in the hull can be computed. The wave exciting force on the platform can be evaluated by integrating the hydrodynamic pressure over the mean wetted hull surface.

\subsubsection{Morison's model}

The hydrodynamic force on slender structures should not be neglected, and can be evaluated by using Morison's equation. The forces acting on slender structures include inertia force and drag force, which are associated with the fluid acceleration and velocity, respectively. Morison's equation is written as

$$
F=\rho V_{M}\left(1+C_{a}\right) \dot{v}+\frac{1}{2} \rho \sigma C_{D} v|v|
$$

where the first term and the second term on the right side denote the inertia force and drag force, respectively; $\rho$ is the water density; $V_{M}$ is displaced volume of a slender element; $v$ and $\dot{v}$ are the horizontal undisturbed fluid velocity and acceleration, respectively; $\sigma$ is the projected area of the element; The added mass $C_{a}$ and drag force coefficients $C_{D}$ are usually determined by calibration with the model test results. In this present study, Morison's equation is only used for drag force, as the inertia forces are computed by using the panel model.

\subsubsection{Mass estimation}

The total weight is divided into four elements, including topside weight, hull steel weight, the weight of ballast water, and remaining weight. The topside weight is kept constant. The hull steel weight, computed by cubic modulus method, is proportional to the hull surface area of SEMI. The remaining element is assumed as constant, and consists of the design margin, fresh water, fuel, and marine load. The total weight is estimated by

$$
W_{\text {Total }}=W_{\text {Topside }}+W_{\text {Steel }}+W_{\text {Ballast }}+W_{\text {Remain }}
$$

where $W_{\text {Total }}$ denotes total weight; $W_{\text {Topside }}$ denotes topside weight; $W_{\text {Steel }}$ denotes hull steel weight; $W_{\text {Ballast }}$ denotes the weight of ballast water.

\subsubsection{Equations of motion}

Through three-dimensional potential flow theory and Morison's equation, added mass, damping coefficient and the wave exciting force can be obtained. By assuming the platform is a rigid body, the equations of motion can be solved in frequency domain

$$
\left[-\omega^{2}(\mathbf{M}+\mathbf{A}(\omega))+i \omega\left(\mathbf{B}_{p}(\omega)+\mathbf{B}_{v}\right)+\mathbf{C}+\mathbf{C}_{e}\right] \boldsymbol{\xi}(\omega, \theta)=\mathbf{F}(\omega, \theta)
$$


Table 1: Wave parameters for survival sea condition

\begin{tabular}{lccccc}
\hline Condition & $H_{s}(m)$ & $T_{p}(s)$ & $\gamma$ & $\sigma_{a}$ & $\sigma_{b}$ \\
\hline Survival & 15.8 & 15.4 & 2.4 & 0.07 & 0.09 \\
\hline
\end{tabular}

where $\boldsymbol{\xi}(\omega, \theta)=\left(\xi_{1}, \xi_{2}, \xi_{3}, \xi_{4}, \xi_{5}, \xi_{6}\right)$ denotes the displacement of rigid body due to incident wave with frequency being $\omega$ and wave heading being $\theta$. The six degrees freedom motion includes translational modes $\boldsymbol{\xi}(\omega, \theta)=$ $\left(\xi_{1}, \xi_{2}, \xi_{3}\right)$ and rotational modes $\boldsymbol{\xi}(\omega, \theta)=\left(\xi_{4}, \xi_{5}, \xi_{6}\right) ; \mathbf{A}(\omega)$ and $\mathbf{B}_{p}(\omega)$ are the added mass and radiation damping matrices, respectively, which are computed by using panel method; $\mathbf{M}$ represents the mass/inertia matrix; $\mathbf{B}_{v}$ denotes the linearized viscous damping matrix, which can be obtained by Morison's model; $\mathbf{C}$ is the hydrostatic restoring matrix; $\mathbf{C}_{e}$ is the external restoring matrix induced by mooring system; $\mathbf{F}(\omega, \theta)$ denotes the first-order wave exciting force.

By solving the equations of motion in frequency domain, the transfer function and phase angle of the motion response in regular waves can be obtained. In the present study, the survival sea state is choosen for analyses, where 100-year hurricane wave condition in central Gulf of Mexico is adopted. This wave condition can be modeled by JONSWAP spectrum, in which the parameters are listed in Table 1. According to the principle of linear superposition, the motion response in irregular waves can be obtained by

$$
S_{r}(\omega, \theta)=|H(\omega, \theta)|^{2} S_{w}(\omega)
$$

where $\theta$ is the wave heading; $H$ denotes the response amplitude operator (RAO). Based on the Rayleigh distribution, the most probable maximum (MPM) response can be computed by

$$
R_{\text {max }}=\sqrt{\left(2 m_{0} \ln \frac{t}{T_{2}}\right)}
$$

where $R_{\max }$ represents the MPM response in $t ; m_{0}$ denotes the zero moment of the response spectrum; $t$ is the time duration and $T_{2}$ is the zero-upcrossing period of the response spectrum.

\subsubsection{Airgap Computations}

Airgap should be evaluated to prevent wave slamming, which may cause structural damage. The airgap due to wave frequency motion and wave runup can be computed by

$$
A G=A G_{\text {static }}-W_{\text {elevation }}+\left(\xi_{3}-\xi_{5} x+\xi_{4} y\right)
$$

where $A G$ represents the airgap; $A G_{\text {static }}$ is the static airgap in calm water, which can be measured from calm water surface to the bottom of the deck. $W_{\text {elevation }}$ represents the wave elevation. $\xi_{3}, \xi_{4}$ and $\xi_{5}$ denote the heave, roll and pitch, respectively. The term $\left(\xi_{3}-\xi_{5} x+\xi_{4} y\right)$ is the vertical combined motion at $(x, y)$. The distribution of probes for estimating the wave elevations on the still water surface $z=0$ and the location of the minimum airgap have been studied by Zhang et al. (2017), and the distribution of wave probes for estimating airgap is illustrated in Figure 3. The MPMin airgap occurs near the northeast column. In the optimization process, the scaled location corresponding to the smallest airgap relative to the column are almost the same. It is because the model shapes are almost the same and the variation in principal dimensions is not significant. 


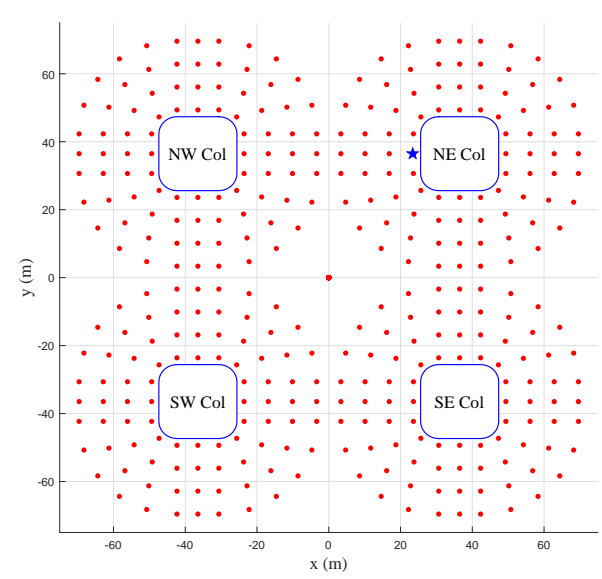

Figure 3: The sketch of the probes (red circles) for estimating the airgap. The blue star symbol represents the probe, which is $2 \mathrm{~m}$ away from the northeast column. This probe is used for airgap analysis in optimization section.

\subsection{Design of experiments}

Design of experiments (DOE), originating from crop cultivation, can reduce test times and get the sample information. The design variables are called factors. There are a number of levels for a factor. At present, the methods for DOE include full factorial design, orthogonal experiment design, and homogeneous design of experiment. It is necessary to apply the most appropriate experimental design strategy. The full factorial design is adopted in the present paper, since it considers different possible combinations of levels across all factors.

Numerical simulations are performed to compute the hydrodynamic performances for each sample point with a specific hull configuration, including the transverse metacentric height, the MPM heave motion, the MPM surge motion, and the MPMin airgap. Meanwhile, the weight of hull is obtained by a mass estimation model.

\subsection{Surrogate model}

The surrogate model, also called as response surface model or approximation model, represents the nonlinear relationship between design variables and their corresponding responses in complex engineering problems by building simple mathematical relationships. In general, numerical simulation is usually required to predict response for a specific design. However, its limitation lies in its complex computations and long computing time. Multi-objective optimization generally requires hundreds or even thousands of iterations to obtain the optimization results. Therefore, it is inefficient to achieve the multi-objective optimization by direct numerical simulations for each varied hull form. Since surrogate model usually relies on simple mathematical models, it can be adopted for rapid prediction of the response for a given design and the computing efficiency can be improved significantly (Audet et al., 2000). Some representative sample points are required for building the surrogate model, which are selected according to DOE. After computing the corresponding response for the selected sample points, the mathematical form can be established for the surrogate model. There are a number of forms for surrogate model, including Response Surface (RS) model, Kriging model, Support Vector Machine (SVM) and Radial Basis Function (RBF). In the present study, RBF is adopted for the surrogate models. 


\subsubsection{Radial basis function}

The RBF can fit nonlinear function and has excellent generalization ability. At present, the generalized RBF neural network has been widely used in the field of data fitting. Buhmann (2009) presented a general discussion of RBF. Zhang et al. (2010) applied the RBF model on seakeeping problem. Generalized RBF neural network is generally categorized into three layers, as illustrated in Figure 4. The squares in the figure represent the input layer. The main function of the input layer is to compute the Euclidean distances between the sample points and the prediction points. The big circles in the figure represent the hidden layer. The function of the hidden layer is to map the input of low-dimensional space to a high-dimensional space through nonlinear function. It is equivalent to finding a surface that best fits training data in an implicit high-dimensional space. The third layer is the output layer, which can get the target output data according to different inputs. The transformation from input space to hidden layer space is nonlinear, while the transformation from hidden layer space to output layer space is linear. This overall mapping process can be written as

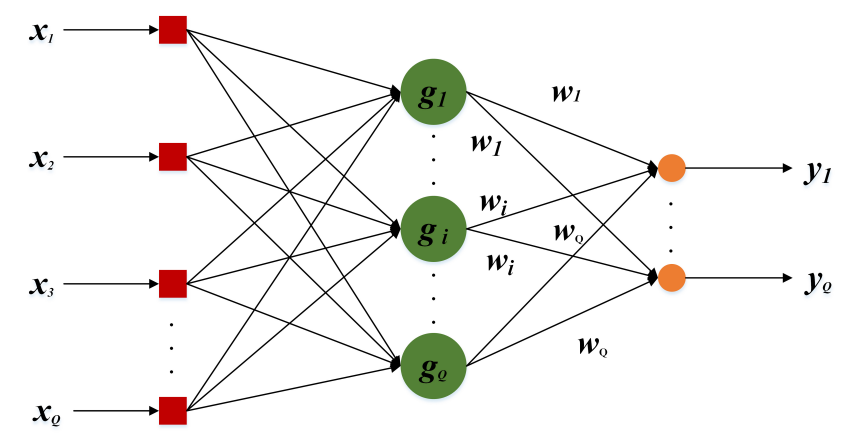

Figure 4: Sketch of RBF surrogate model. Design variables: $\left[\boldsymbol{x}_{1}, \boldsymbol{x}_{2}, \ldots, \boldsymbol{x}_{Q}\right]$; objective values: $\left[\boldsymbol{y}_{1}, \ldots, \boldsymbol{y}_{Q}\right]$; radial basis functions: $\left[\boldsymbol{g}_{1}, \ldots, \boldsymbol{g}_{i}, \ldots, \boldsymbol{g}_{Q}\right]$; weights: $\left[\boldsymbol{w}_{1}, \ldots, \boldsymbol{w}_{i}, \ldots, \boldsymbol{w}_{Q}\right]$. Squares: input layer; big circle nodes: hidden layer; small circle nodes: output layer.

$$
\begin{gathered}
g_{j}(\boldsymbol{x})=g\left(\left\|\boldsymbol{x}-\boldsymbol{x}_{j}\right\|\right), \quad j=1, \ldots, Q \\
\boldsymbol{y}_{i}=\sum_{j=1}^{Q} \boldsymbol{w}_{j} g_{j}\left(\boldsymbol{x}_{i}\right)
\end{gathered}
$$

where $\boldsymbol{x}$ is the vector of interpolation points; $\boldsymbol{x}_{j}$ is the vector of the $j$ th sample points; $Q$ denotes the number of sample points. The approximating function $\boldsymbol{y}_{i}$ is represented as a sum of $Q$ radial basis functions $g_{j}\left(\boldsymbol{x}_{i}\right)$, each associated with different center positions $\boldsymbol{x}_{j}$, and weighted by an appropriate coefficient $\boldsymbol{w}_{j}$.

Gaussian, Cubic, Inverse Multi-Quadric (IMQ) and Multi-Quadric (MQ) are the commonly used RBFs. Since IMQ RBF has been widely used in engineering, and a lot of valuable engineering experience can be referred to, we apply IMQ radial basis function, which can be written as

$$
g_{j}(\boldsymbol{x})=\frac{1}{\sqrt{\left\|\boldsymbol{x}-\boldsymbol{x}_{j}\right\|^{2}+b^{2}}}
$$

where $b$ is formal parameter. The value of formal parameter $b$ should be chosen properly, since it has significant impact on the accuracy of the surrogate model. As one of the most widely used approaches, the LOOCV is adopted to find the best formal parameters $b$ in the present paper. This process is shown in Figure 5 . 


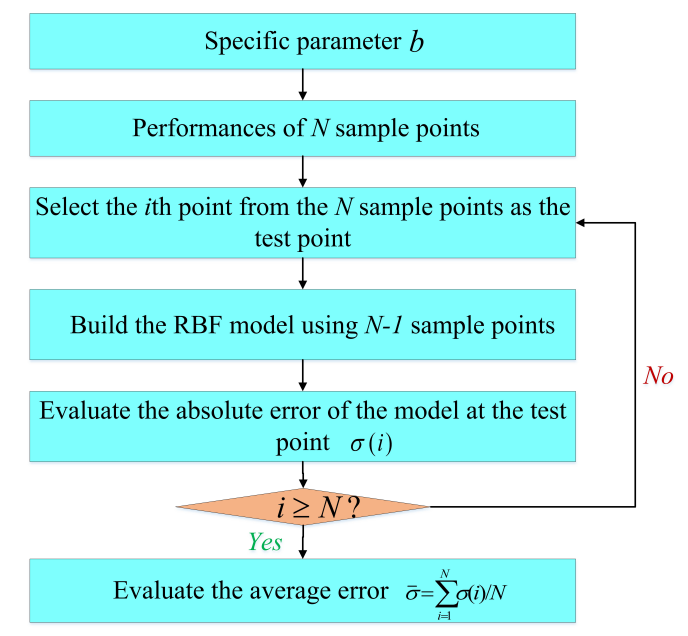

Figure 5: The flow chart of the leave-one-out cross validation.

\subsection{Multi-objective optimization}

Multi-objective optimization has been applied in many fields, where optimal decisions need to be taken in the presence of trade-offs between two or more conflicting objectives. For example, designers often search for design parameters to improve product quality while reducing the costs.

In general, there are two methods to solve multi-objective optimization problems. The first one is indirect method. That is to say, multiple objectives of the problem are transformed into a new objective function through the allocation of weight coefficients, so that the single objective optimization method can be used to solve the problem. The other method is to directly solve the multi-objective optimization problem and the objective functions remain unchanged in the optimization process. Since the objective functions conflict with each other in most scenarios, a number of Pareto-optimal solutions can be obtained by optimization. These Pareto-optimal solutions are also called non-dominated solutions or non-inferior solutions, which means none of the objective functions can be improved without degrading the other objectives. From different viewpoints and goals, various results may be obtained for the multi-objective optimization. These results may be a representative set of Pareto optimal solutions.

\subsubsection{Multi-Objective Particle Swarm Optimization}

Particle swarm optimization (PSO) is a kind of evolutionary technology based on swarm intelligence that simulates social behavior. Its key idea is built on the collaboration and information sharing between the individuals in the group (Eberhart and Kennedy, 1995). Particle is attracted toward the location of the current global best position $X_{g b e s t}$ and its own best position $X_{\text {pbest }}$ (Yang, 2010).

The basic rules of the PSO algorithm can be explained in three main stages. First, the fitness value of each particle is evaluated. Then, by comparing each particle fitness value with the current particle and the overall particles, the local best position $X_{\text {pbest }}$ and the global best position $X_{\text {gbest }}$ are detected. Finally, the velocity and the position of each particle are updated. The flowchart of the algorithm is illustrated in Figure 6.

Reyes-Sierra and Coello Coello (2006) proposed Multi-Objective Particle Swarm Optimization (MOPSO). Just like PSO, particles in MOPSO share information and move towards their own personal (local) best memory 


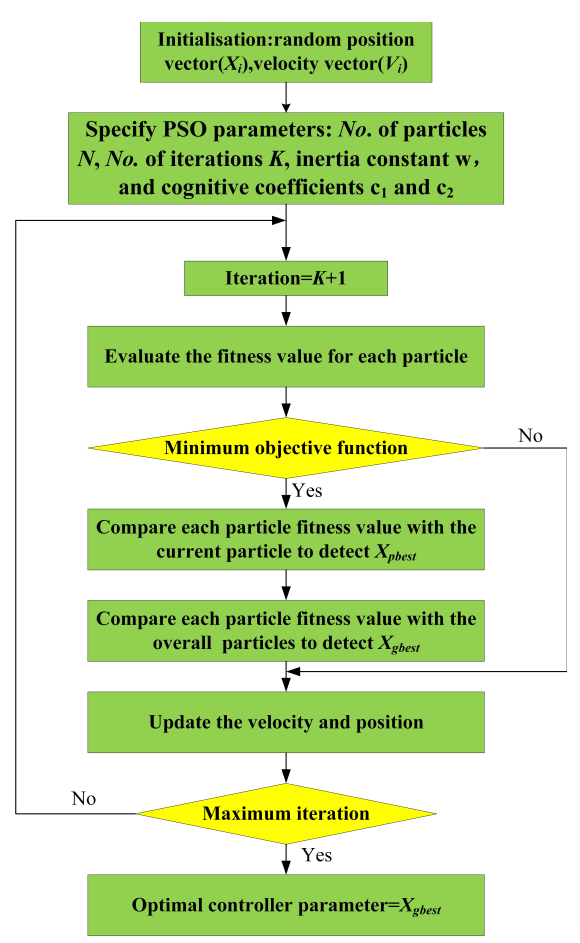

Figure 6: The description of the principle for Particle Swarm Optimization (PSO).

and global best particles. However, unlike PSO, there are more than one criterion to determine the local or global best particle. The concepts of non-dominated sorting are introduced into MOPSO. All of the non-dominated particles in the swarm are gathered into a sub-swarm called repository. A leader of particles is selected in repository. Particle in less crowded regions has more chance to be selected as a leader (Santana et al., 2009).

\subsubsection{Objectives and constraints for optimization}

Usually, the methods to handle the constraints include rejecting infeasible solutions, penalty function methods, and various correction algorithms. Penalty function method is the most classic and convenient method to deal with the constraints. The idea of the penalty function method is to combine the objective function with the constraints to form a new objective function. In the present study, penalty function method is adopted and can be written as

$$
\begin{array}{rr}
\min & f(\mathbf{x})=\left[f_{1}, f_{2}, \ldots, f_{m}\right] \\
& \mathbf{x}=\left[x_{1}, x_{2}, \ldots, x_{n}\right] \\
\text { s.t. } & g_{i}(\mathbf{x}) \geqslant 0, \quad i=1, \ldots, k \\
& h_{j}(\mathbf{x})=0, \quad j=1, \ldots, l
\end{array}
$$

where the function $f$ is the $m$-dimensional multi-objective function. $\mathbf{x}$ denotes design variable with an $n$ dimensional array. The $g_{i}$ and $h_{j}$ represent the $i$ th inequality constraint and $j$ th equation constraint, respectively. Constraints can be expressed as penalty functions, which can be written as

$$
P(\mathbf{x})=\sum_{i=1}^{k} \max \left[0,-g_{i}(\mathbf{x})\right]^{2}+\sum_{j=1}^{l}\left|h_{j}(\mathbf{x})\right|^{2}
$$


when $\mathbf{x}$ fails to satisfy one of the constraints, the penalty function will be a positive value. In this way, the constrained optimization problem transforms the objective functions as

$$
\min F(\mathbf{x}, \sigma)=f(\mathbf{x})+\sigma P(\mathbf{x})
$$

where $\sigma$ is penalty factor defined as a very large positive number. In the optimization, if one of the constraints is not fulfilled, the second term on the right side of Eqn.(20) will be very large, which means the solution will be excluded.

In the present study, the MPM heave motion and the total weight of the platform are selected as the two objectives. The MPM heave and total structural weight represent the safety and cost, respectively. The hull forms should be optimized for higher safety and lower cost. Three constraints are the transverse metacentric height, the MPM surge motion and the MPMin airgap.

\section{Case studies}

Detailed analyses and computations have been performed for three types of SEMIs including FPU, HVS and FDU.

\subsection{Semi-submersible floating production unit}

The sketch of SEMI FPU is illustrated in Figure 7. The definition of the incident wave heading is shown

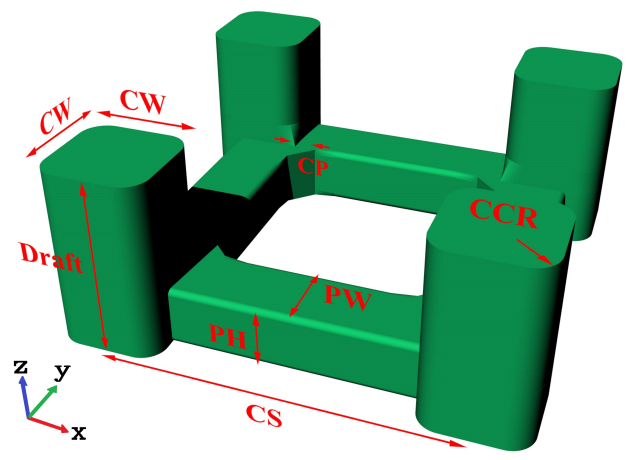

Figure 7: The sketch of SEMI FPU. CW: column width; CS: column spacing; PW: pontoon width; PH: pontoon height; CP: width of cakepiece; CCR: column corner radius.

in Figure 8, where a plan view of the platform is presented. The main particulars of the initial design are listed in Table 2.

\subsubsection{Surrogate model}

In the present study, full factorial design is adopted. The upper and lower bounds of different factors, and level steps of all factors are listed in Table 3. Once the level number is determined, the level step can be computed by

$$
\text { Level step }=(\text { Upper bound }- \text { Lower bound }) /(\text { Level number }-1)
$$




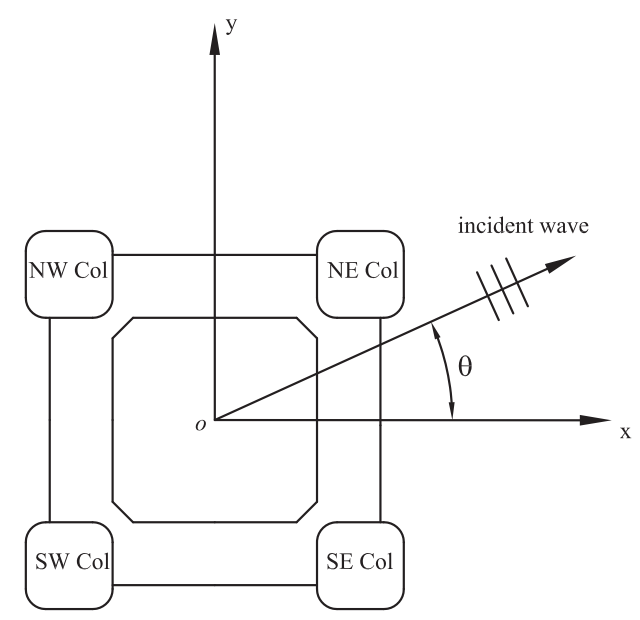

Figure 8: Definition of incident wave heading $\theta$ (plan view) and index of the four columns. NW Col: northwest column; NE Col: northeast column; SW Col: southwest column; SE Col: southeast column.

Table 2: Main particulars of the initial SEMI FPU

\begin{tabular}{|c|c|c|c|}
\hline Description & Unit & Symbol & Value \\
\hline Length & $\mathrm{m}$ & $\mathrm{L}$ & 94.72 \\
\hline Draft & $\mathrm{m}$ & Draft & 31.0 \\
\hline Freeboard & $\mathrm{m}$ & FB & 20.0 \\
\hline Number of columns & / & / & 4 \\
\hline Column width & $\mathrm{m}$ & $\mathrm{CW}$ & 21.76 \\
\hline Column length & $\mathrm{m}$ & $\mathrm{CL}$ & 21.76 \\
\hline Column spacing (center to center) & $\mathrm{m}$ & CS & 72.96 \\
\hline Column corner radius & $\mathrm{m}$ & $\mathrm{CCR}$ & 5.045 \\
\hline Pontoon width & $\mathrm{m}$ & $\mathrm{PW}$ & 21.76 \\
\hline Pontoon height & $\mathrm{m}$ & $\mathrm{PH}$ & 10.88 \\
\hline Pontoon corner radius & $\mathrm{m}$ & PCR & 1.28 \\
\hline Width of cakepiece & $\mathrm{m}$ & $\mathrm{CP}$ & 5.12 \\
\hline Displacement & metric ton & $\Delta$ & 108,031 \\
\hline Transverse metacentric height & $\mathrm{m}$ & $G M_{T}$ & 6.0 \\
\hline Longitudinal metacentric height & $\mathrm{m}$ & $G M_{L}$ & 6.0 \\
\hline
\end{tabular}

Table 3: Full factorial design for SEMI FPU.

\begin{tabular}{cccccc}
\hline Factors & Lower bound & Initial & Upper bound & Level number & Level step \\
\hline Draft & $27.9 \mathrm{~m}$ & $31 \mathrm{~m}$ & $34.1 \mathrm{~m}$ & 5 & $1.55 \mathrm{~m}$ \\
Column spacing & $65.664 \mathrm{~m}$ & $72.96 \mathrm{~m}$ & $80.256 \mathrm{~m}$ & 5 & $3.648 \mathrm{~m}$ \\
Column width & $19.584 \mathrm{~m}$ & $21.76 \mathrm{~m}$ & $23.936 \mathrm{~m}$ & 3 & $2.176 \mathrm{~m}$ \\
Pontoon hight & $9.792 \mathrm{~m}$ & $10.88 \mathrm{~m}$ & $11.968 \mathrm{~m}$ & 3 & $1.088 \mathrm{~m}$ \\
Width of cakepiece & $4.608 \mathrm{~m}$ & $5.12 \mathrm{~m}$ & $5.632 \mathrm{~m}$ & 3 & $0.512 \mathrm{~m}$ \\
\hline
\end{tabular}


Based on the permutation and combination theory of the factors with different levels, totally $5^{2} \times 3^{3}=675$ sample points are selected for the surrogate models. In order to ensure the accuracy of the surrogate models, the LOOCV is adopted to find the best formal parameters $b$. The average errors of the surrogate models with different formal parameters are illustrated in Figure 9.
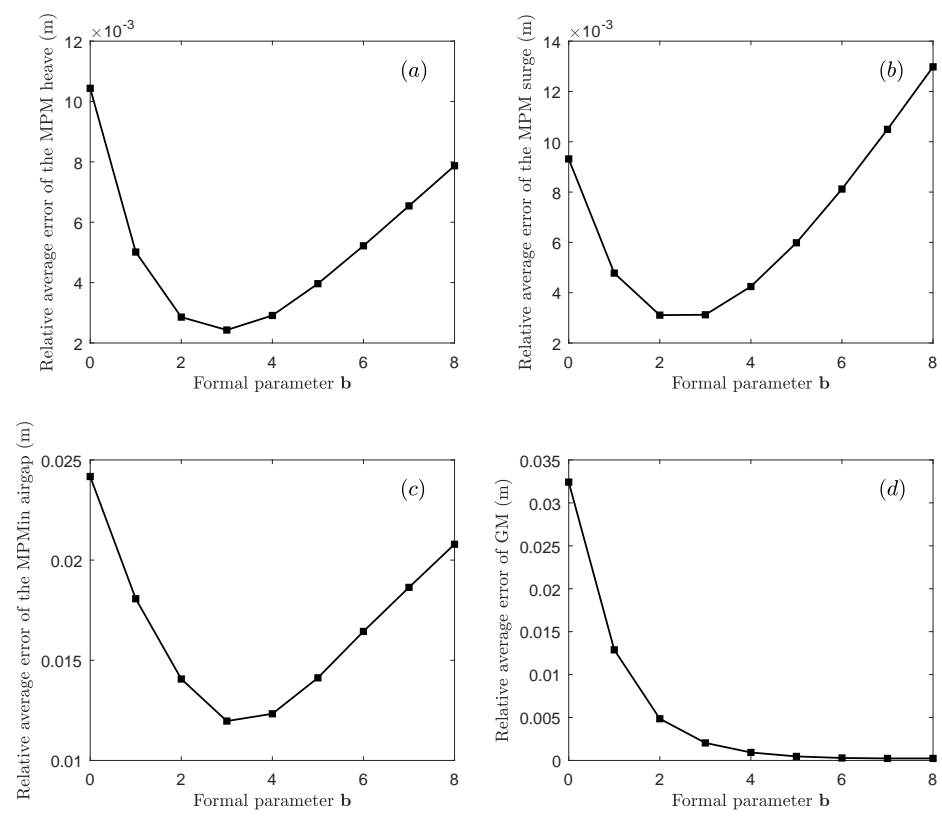

Figure 9: The average errors of RBF models by LOOCV with respect to the formal parameter for SEMI FPU. (a) The MPM heave motion; (b) The MPM surge motion; $(c)$ The MPMin airgap; $(d)$ Transverse metacentric height. JONSWAP spectrum $H_{s}=15.8$ $\mathrm{m} ; T_{p}=15.4 \mathrm{sec} ;$ peak enhancement factor $\gamma=2.4$.

Figure 9 shows that the best formal parameters $b$ of RBF models are 3, 3, 3 and 7 for heave, surge, airgap, and transverse metacentric height, respectively. As shown in Figure 10, the surrogate models with specific parameters are validated by comparing with the direct numerical simulations. After determining the formal parameters of different RBF models, the MPM heave motion response, the MPM surge motion response, the MPMin airgap, and transverse metacentric height can be rapidly estimated by using the surrogate models. In order to get the correlations between the single design variable of hull sizing and the MPM heave motion response, the variation tendencies of the MPM heave motion response are obtained by varying single design variable while the others are kept unchanged. As illustrated in Figure 11, when the draft increases, the MPM heave motion will reduce. However, when the column spacing, column width, pontoon height, or width of cakepiece gradually decreases, the MPM heave motion will reduce.

\subsubsection{Pareto-optimal solutions}

MOPSO is employed for the optimization, in which the population size and maximum generation are both set to 100. Finally, non-dominated 30 Pareto-optimal solutions are obtained. The Pareto-optimal solutions are ranked with the increase of heave motion, as listed in Table 4. The MPM heave motion and total weight can be reduced by up to $12.68 \%$ and $12.16 \%$, respectively. The results of the optimization are shown in Figure 12 . 

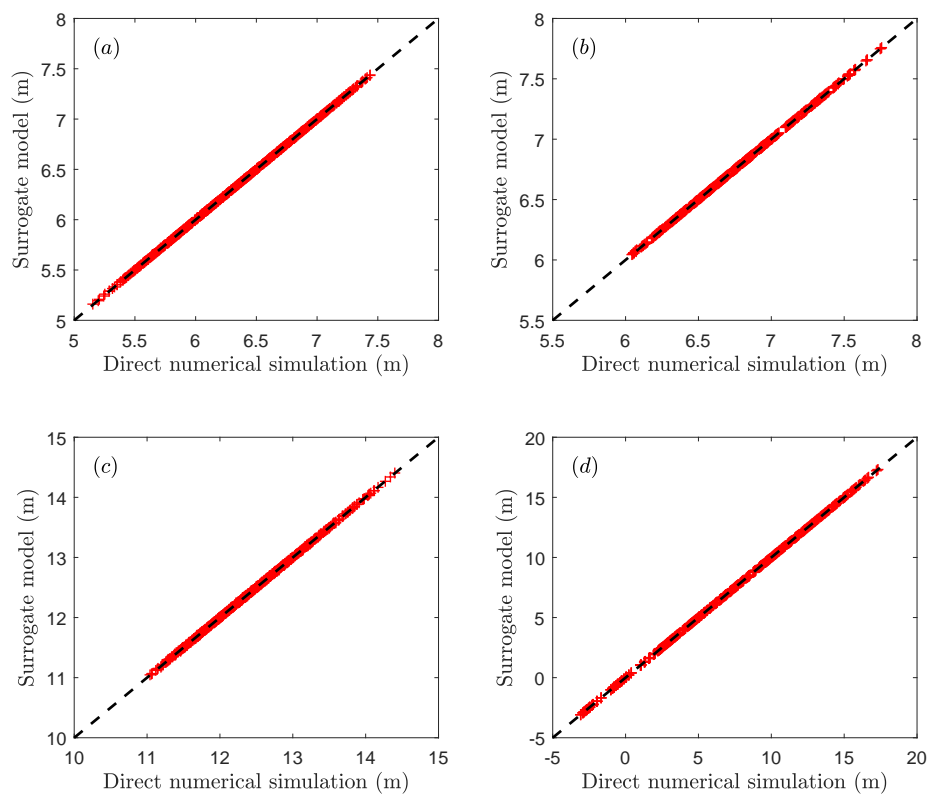

Figure 10: Comparison of the surrogate models with specific parameters and the direct numerical simulations. (a) The MPM heave motion $(b=3) ;(b)$ The MPM surge motion $(b=3) ;(c)$ The MPMin airgap $(b=3) ;(d)$ Transverse metacentric height $(b=7)$.

The rhombus symbol in the plot represents the two objective values for the initial hull design. Through the vertical and horizontal axes passing the initial point, the plot is divided into four regions. In the present study, the 29 Pareto-optimal solutions, which are located in bottom left region, are better than the initial design in terms of both objectives. Only 1 Pareto-optimal solution is worse than the initial design in MPM heave motion. The MPM heave motion of this hull form is increased by $1.16 \%$, but its total weight is reduced by $12.16 \%$. As shown in Figure 13, by comparing the results of surrogate models with the direct numerical simulations, it is found that the maximum relative error is less than $2 \%$, which further confirms the accuracy of the present surrogate model. Moreover, the MPM surge motion, the MPMin airgap, and the transverse metacentric height for the 30 Pareto-optimal solutions are all computed, and illustrated in Figure 14. As shown, the obtained 30 Pareto-optimal solutions fulfill the constraints.

According to the 30 Pareto-optimal solutions obtained by optimization, the variation tendencies of five design variables are illustrated in Figure 15. The $x$-axis represents the sequence number of the Pareto-optimal solution, and the $y$-axis represents the design variables. As the sequence number of Pareto-optimal solutions increases, the total weight decreases while the MPM heave motion increases. As shown in Figure 15 (a), increasing draft can help reduce the heave motion but increase the total weight. The effect of draft on MPM heave motion is consistent with the previous analyses shown in Figure $11(a)$. The column spacing values are around the upper bound among 30 Pareto-optimal solutions, and the column width declines from $21 \mathrm{~m}$ to $20 \mathrm{~m}$, which can be observed in Figures $15(b)$ and $(c)$. The pontoon heights and cakepiece widths of the 30 Pareto-optimal solutions are illustrated in Figures $15(d)$ and $(e)$, respectively. The pontoon height values are around the lower bound, and the widths of cakepiece rise from $4.6 \mathrm{~m}$ to $5.2 \mathrm{~m}$. In order to validate the optimization results, heave RAO of the initial SEMI FPU and case No.11 Pareto-optimal solutions are 

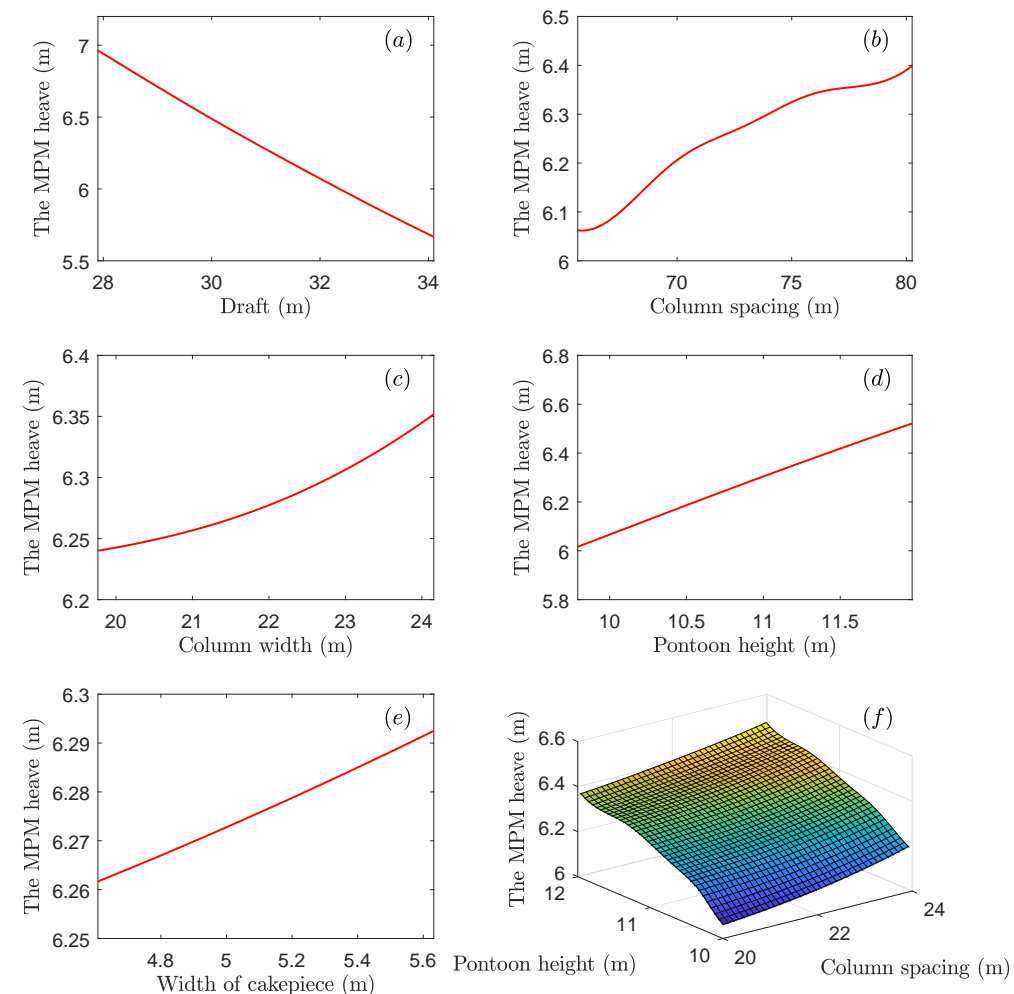

Figure 11: The correlations between the single design variable and the MPM heave motion response. (a) Draft; (b) Column spacing; (c) Column width; (d) Pontoon height; (e) Width of cakepiece; $(f)$ Column spacing and Pontoon height.

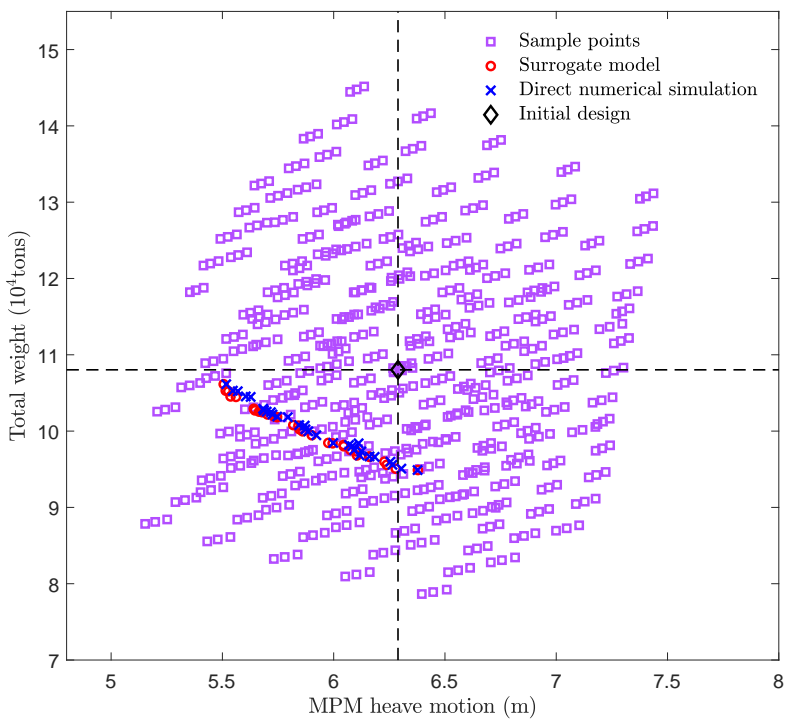

Figure 12: 30 Pareto-optimal solutions for SEMI FPU obtained by MOPSO based on the RBF model and compared with the direct numerical simulations. 


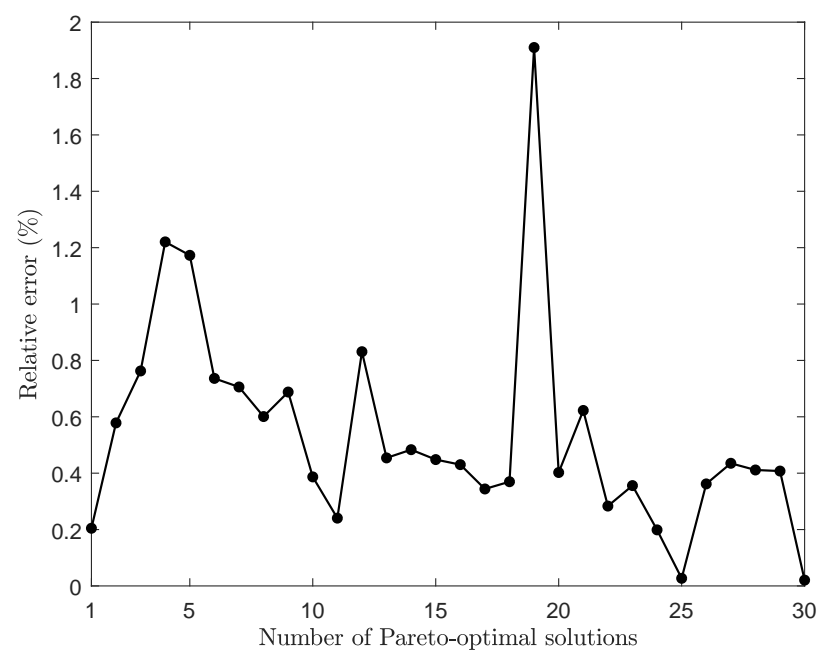

Figure 13: The relative error for SEMI FPU between the predictions using surrogate models and direct numerical simulations.
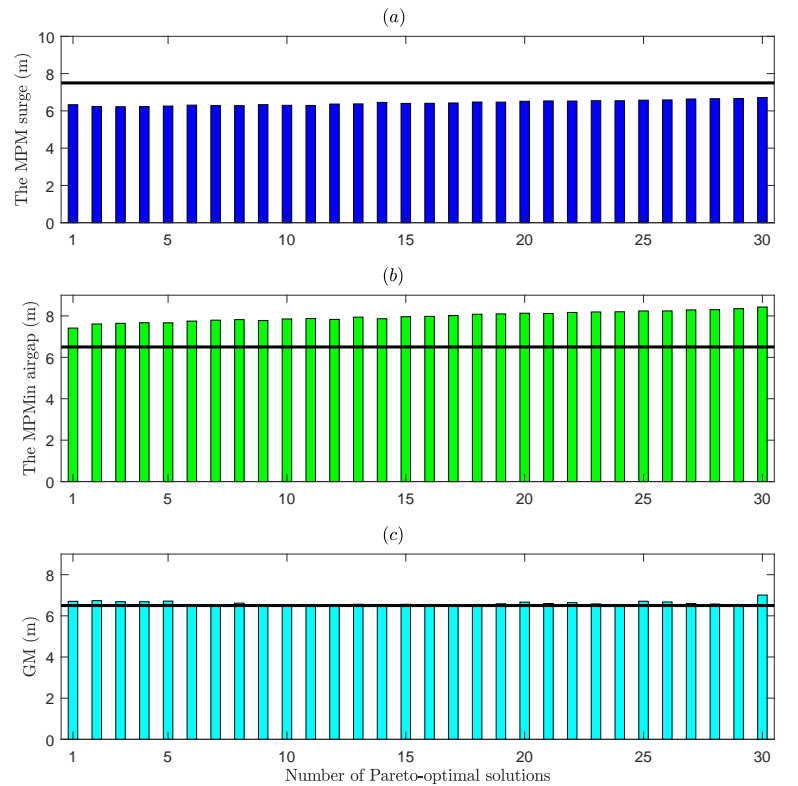

Figure 14: The constraint values of 30 Pareto-optimal solutions for SEMI FPU. (a) The MPM surge motion should be less than $7.5 \mathrm{~m}$; (b) The MPMin airgap should be greater than $6.5 \mathrm{~m}$; (c) Transverse metacentric height should be larger than $6.5 \mathrm{~m}$. 
Table 4: The Pareto-optimal solutions for SEMI FPU obtained by utilizing MOPSO.

\begin{tabular}{|c|c|c|c|c|c|c|c|c|c|}
\hline & Draft & CS & $\mathrm{CW}$ & $\mathrm{PH}$ & $\mathrm{CP}$ & Heave $_{\text {MPM }}$ & Percentage & Weight & Percentage \\
\hline Unit & $\mathrm{m}$ & $\mathrm{m}$ & $\mathrm{m}$ & $\mathrm{m}$ & $\mathrm{m}$ & $\mathrm{m}$ & $\%$ & $10^{4}$ ton & $\%$ \\
\hline Initial & 31.000 & 72.960 & 21.760 & 10.880 & 5.120 & 6.305 & 0.000 & 10.803 & 0.000 \\
\hline 1 & 34.100 & 77.193 & 20.888 & 9.792 & 4.643 & 5.506 & -12.677 & 10.614 & -1.746 \\
\hline 2 & 34.100 & 78.717 & 20.583 & 9.808 & 4.773 & 5.515 & -12.531 & 10.530 & -2.531 \\
\hline 3 & 34.100 & 78.933 & 20.524 & 9.839 & 4.880 & 5.527 & -12.347 & 10.522 & -2.603 \\
\hline 4 & 33.987 & 79.111 & 20.470 & 9.792 & 4.794 & 5.537 & -12.187 & 10.454 & -3.230 \\
\hline 5 & 33.819 & 78.797 & 20.533 & 9.792 & 4.889 & 5.561 & -11.800 & 10.448 & -3.288 \\
\hline 6 & 33.351 & 78.797 & 20.424 & 9.792 & 4.844 & 5.641 & -10.529 & 10.293 & -4.725 \\
\hline 7 & 33.397 & 79.336 & 20.319 & 9.792 & 4.934 & 5.646 & -10.454 & 10.271 & -4.922 \\
\hline 8 & 33.353 & 79.686 & 20.267 & 9.792 & 4.872 & 5.665 & -10.155 & 10.255 & -5.076 \\
\hline 9 & 33.150 & 78.737 & 20.411 & 9.792 & 4.922 & 5.679 & -9.934 & 10.248 & -5.143 \\
\hline 10 & 33.175 & 79.583 & 20.236 & 9.833 & 4.926 & 5.703 & -9.550 & 10.218 & -5.419 \\
\hline 11 & 33.198 & 80.197 & 20.107 & 9.857 & 4.848 & 5.732 & -9.085 & 10.190 & -5.675 \\
\hline 12 & 32.810 & 78.797 & 20.374 & 9.817 & 4.849 & 5.746 & -8.868 & 10.183 & -5.741 \\
\hline 13 & 32.534 & 79.563 & 20.193 & 9.822 & 4.932 & 5.818 & -7.717 & 10.079 & -6.706 \\
\hline 14 & 32.261 & 78.345 & 20.405 & 9.798 & 4.940 & 5.842 & -7.349 & 10.071 & -6.773 \\
\hline 15 & 32.338 & 79.477 & 20.189 & 9.792 & 4.999 & 5.847 & -7.269 & 10.024 & -7.214 \\
\hline 16 & 32.277 & 79.578 & 20.152 & 9.792 & 4.981 & 5.861 & -7.047 & 9.997 & -7.463 \\
\hline 17 & 32.098 & 79.743 & 20.091 & 9.804 & 4.969 & 5.902 & -6.398 & 9.946 & -7.937 \\
\hline 18 & 31.659 & 79.631 & 20.064 & 9.792 & 5.054 & 5.976 & -5.221 & 9.845 & -8.864 \\
\hline 19 & 31.669 & 80.087 & 19.995 & 9.795 & 5.120 & 5.995 & -4.913 & 9.840 & -8.918 \\
\hline 20 & 31.291 & 79.567 & 20.106 & 9.807 & 5.071 & 6.045 & -4.130 & 9.819 & -9.114 \\
\hline 21 & 31.226 & 79.229 & 20.146 & 9.792 & 5.095 & 6.045 & -4.122 & 9.801 & -9.275 \\
\hline 22 & 31.161 & 79.878 & 20.013 & 9.792 & 5.056 & 6.075 & -3.652 & 9.753 & -9.723 \\
\hline 23 & 31.014 & 79.694 & 20.018 & 9.820 & 5.112 & 6.102 & -3.217 & 9.734 & -9.900 \\
\hline 24 & 31.038 & 80.073 & 19.910 & 9.793 & 5.055 & 6.105 & -3.175 & 9.681 & -10.387 \\
\hline 25 & 30.726 & 79.965 & 19.974 & 9.792 & 5.114 & 6.159 & -2.310 & 9.668 & -10.507 \\
\hline 26 & 30.657 & 79.690 & 20.015 & 9.792 & 5.097 & 6.162 & -2.274 & 9.662 & -10.564 \\
\hline 27 & 30.291 & 79.345 & 20.026 & 9.822 & 5.105 & 6.228 & -1.225 & 9.599 & -11.143 \\
\hline 28 & 30.199 & 79.319 & 20.010 & 9.792 & 5.124 & 6.238 & -1.059 & 9.558 & -11.521 \\
\hline 29 & 30.044 & 79.559 & 19.923 & 9.814 & 5.141 & 6.278 & -0.423 & 9.508 & -11.989 \\
\hline 30 & 29.543 & 79.779 & 20.003 & 9.792 & 5.163 & 6.378 & 1.163 & 9.489 & -12.160 \\
\hline
\end{tabular}

compared and illustrated in Figure 16. The wave spectrum of the survival condition is also plotted in Figure 16. As presented, most of the wave energy concentrates around $15.4 \mathrm{~s}$, where the hump of heave RAO for optimal solution is smaller than the initial design. The CFD tool STAR-CCM+ is also adopted to evaluate the heave motion of the platforms, where VOF method and $k-\varepsilon$ turbulence model are applied. The total number of mesh cells is around 2 million. As shown in Figure 16, the CFD simulations for the initial and optimal designs have been carried out at the same incoming wave periods around the hump region. Results of the proposed model by combining panel method and Morison's equation agree well with CFD simulations for wave periods smaller than 24 sec. Since the heave RAOs for wave period being larger than $24 \mathrm{sec}$ have negligible effects on heave MPM (for a JONSWAP wave spectrum with Tp being around $15 \mathrm{sec}$ ), the accuracy of the panel method and Morison's equation is acceptable. As shown in Figure 16, the results obtained from panel method and Morison's 

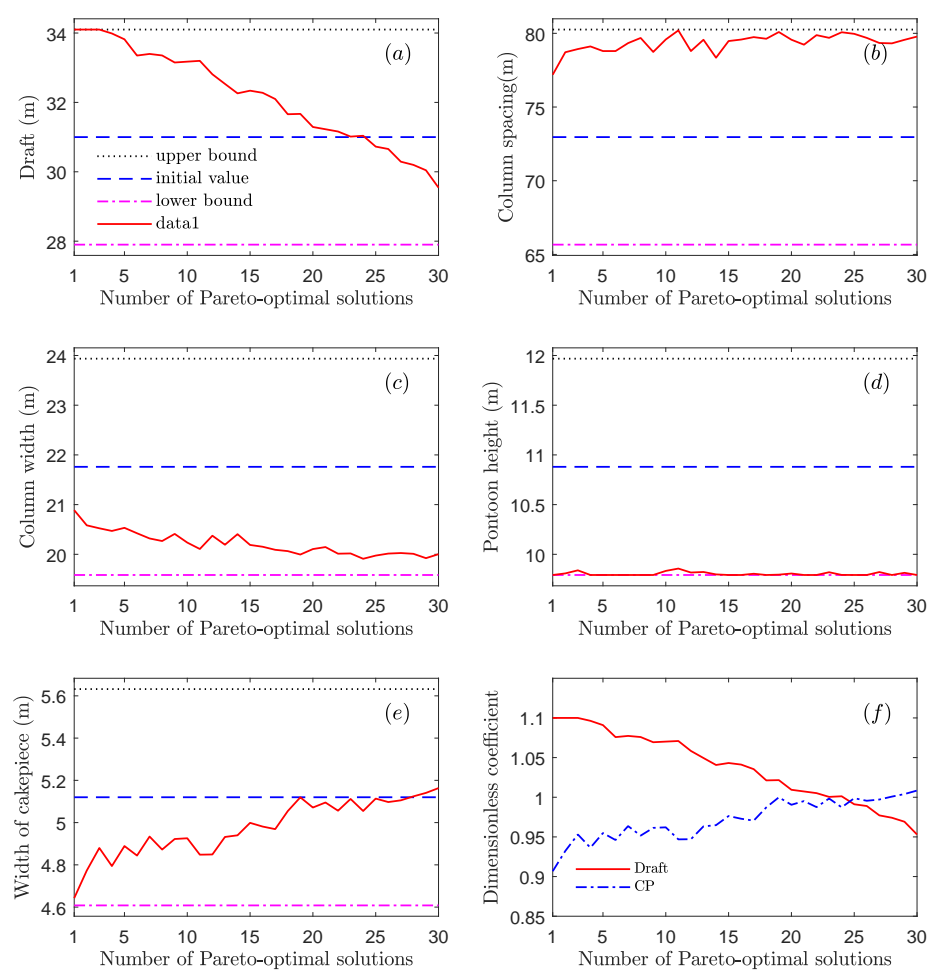

Figure 15: The variation of five design variables with respect to 30 Pareto-optimal solutions for SEMI FPU. (a) Draft; (b) Column spacing; $(c)$ Column width; $(d)$ Pontoon height; $(e)$ Width of cakepiece; $(f)$ Non-dimensional draft and width of cakepiece.

equation are in the same trend with those obtained by using CFD tool for both initial and Case No.11 optimal designs, so that the panel method and Morison's equation can be used for building the surrogate model.

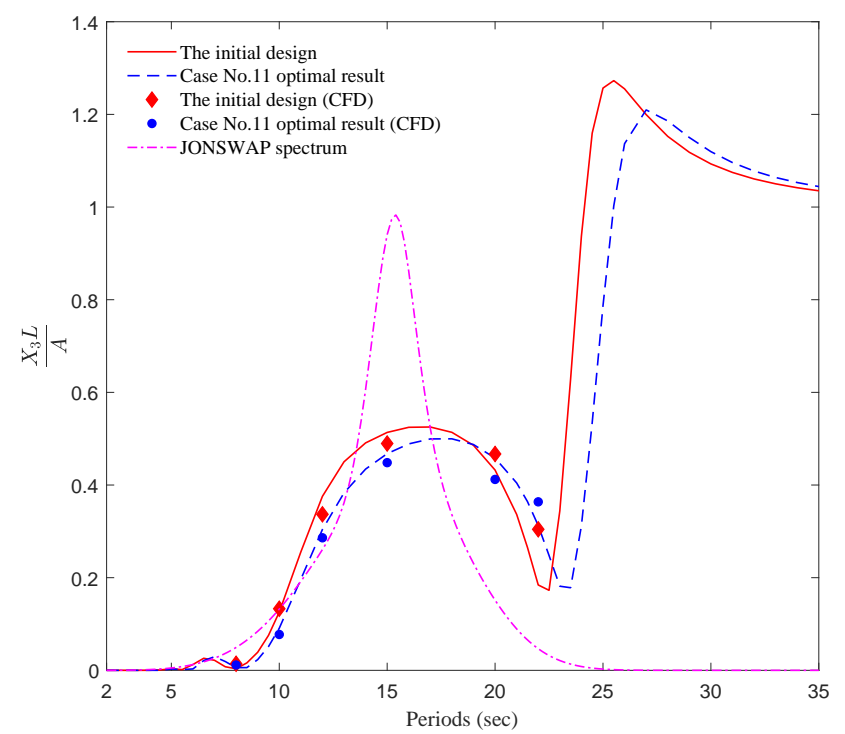

Figure 16: Heave RAOs of one optimal design and the initial SEMI FPU design. JONSWAP spectrum $H_{s}=15.8 \mathrm{~m} ; T_{p}=15.4$ sec; peak enhancement factor $\gamma=2.4$. $A$ is the amplitude of incident wave. $L$ is the characteristic length of the SEMI. 
Based on the multi-objective optimization for the SEMI FPU, it is found that when the draft of platform increases, the MPM heave motion will reduce. However, when the column spacing, column width, pontoon height, or width of cakepiece gradually decreases, the MPM heave motion will reduce. From the 30 Paretooptimal solutions, it can be observed that the MPM heave motion and total weight can be reduced by $12.68 \%$ and $12.16 \%$ at most, respectively. Increasing draft and column spacing should be considered as a priority in order to ensure safety. It is suggested that the column spacing should be retained around the upper bound of the design. Moreover, the pontoon height and column width should be sized as small as possible to achieve better motion and less weight.

\subsection{Heave and VIM Suppressed Semi-submersible}

Recently, HVS semi-submersible has been developed as a new concept, which could be used for wet-tree or dry-tree application. The lower part of each column has been wrapped by a blister, which redistributes displacement of pontoons and the base of the columns. Due to this arrangement, the heave motion and VIM may be suppressed (Kyoung et al., 2015). Because of the blisters attached to the columns, the coherence of vortex shedding can be broken (Xu, 2011). The effective column excitation length is computed by

$$
\text { The effective column excitation length }=\text { Column draft }- \text { Blister height }
$$

Since the draft ranges from $27.9 \mathrm{~m}$ to $34.1 \mathrm{~m}$ and the blister height is $12 \mathrm{~m}$ during the optimization process, the effective column excitation length is between $15.9 \mathrm{~m}$ and $22.1 \mathrm{~m}$. Due to the blisters attached to the columns, the effective column excitation length is reduced by about $40 \%$. Thus, this semi-submersible experiences much less or no VIM. The sketch of a typical HVS semi-submersible is illustrated in Figure 17. The main particulars

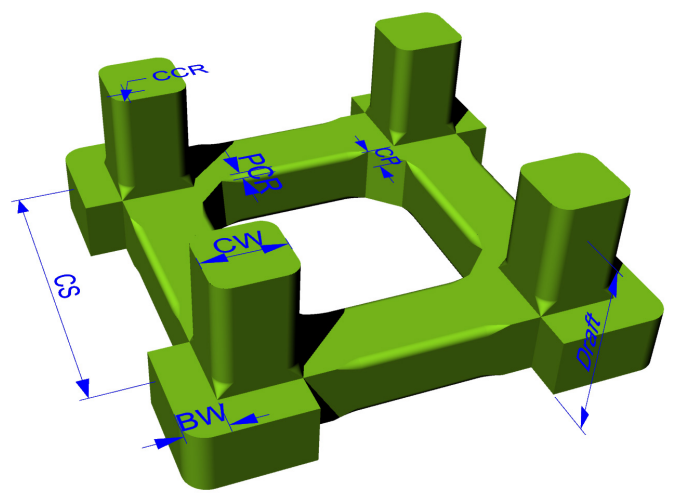

Figure 17: Definition of the main particulars of the base HVS semi-submersible. CW: column width; CS: column spacing; CP: width of cakepiece; CCR: column corner radius; PCR: pontoon corner radius; BW: blister width. [Xu (2011)]

of the initial design are listed in Table 5. The contour of airgaps for HVS semi-submersible in long-crested irregular waves is shown in Figure 18. As shown, the MPMin airgap is $2.53 \mathrm{~m}$ and occurs at the point $(23.4 \mathrm{~m}$, $36.48 \mathrm{~m}, 0 \mathrm{~m}$ ), which is located at the left side of NE column in wave heading $\theta=0^{\circ}$.

\subsubsection{Surrogate model}

The upper and lower bounds of different factors, and level steps of all factors are listed in Table 6 . Totally $3^{6}=729$ sample points are selected for the surrogate models. 
Table 5: Main particulars of the initial HVS semi-submersible

\begin{tabular}{lccc}
\hline Description & Unit & Symbol & Value \\
\hline Length & $\mathrm{m}$ & $\mathrm{L}$ & 93 \\
Draft & $\mathrm{m}$ & Draft & 31.0 \\
Freeboard & $\mathrm{m}$ & $\mathrm{FB}$ & 20.0 \\
Number of columns & $/$ & $/$ & 4 \\
Column width & $\mathrm{m}$ & $\mathrm{CW}$ & 20 \\
Column length & $\mathrm{m}$ & $\mathrm{CL}$ & 20 \\
Column spacing (center to center) & $\mathrm{m}$ & $\mathrm{CS}$ & 73 \\
Column corner radius & $\mathrm{m}$ & $\mathrm{CCR}$ & 4 \\
Pontoon width & $\mathrm{m}$ & $\mathrm{PW}$ & 20 \\
Pontoon height & $\mathrm{m}$ & $\mathrm{PH}$ & 12 \\
Pontoon corner radius & $\mathrm{m}$ & $\mathrm{PCR}$ & 1.28 \\
Width of cakepiece & $\mathrm{m}$ & $\mathrm{CP}$ & 8 \\
Blister width & $\mathrm{m}$ & $\mathrm{BW}$ & 7.5 \\
Blister corner radius & $\mathrm{m}$ & $\mathrm{BCR}$ & 4 \\
Displacement & $\mathrm{m}$ & $G M_{L}$ & 6.5 \\
Transverse metacentric height & $\mathrm{m}$ & $\Delta$ & 122,030 \\
Longitudinal metacentric height & $\mathrm{m}$ & 6.5 \\
\hline
\end{tabular}
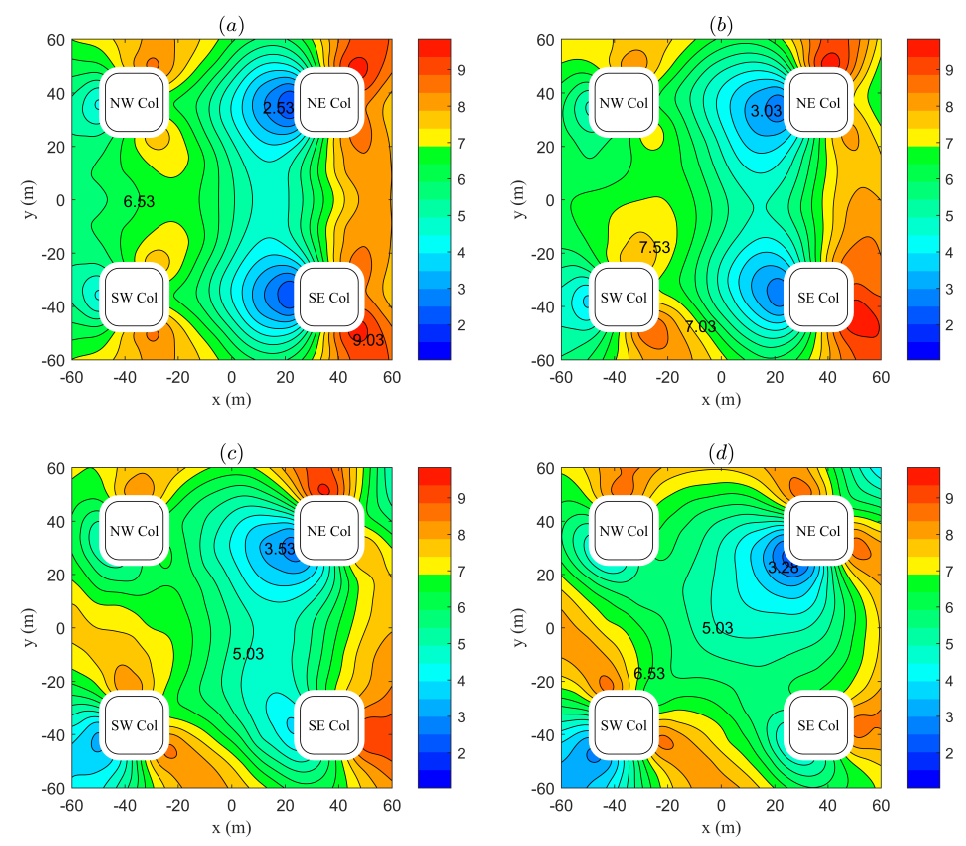

Figure 18: Contour of airgap for HVS semi-submersible in long-crested irregular waves, $H_{s}=15.8 \mathrm{~m} ; T_{p}=15.4 \mathrm{~s} ;$ peak enhancement factor $\gamma=2.4$. (a) $\theta=0^{\circ} ;(b) \theta=15^{\circ} ;(c) \theta=30^{\circ} ;(d) \theta=45^{\circ}$.

The prediction errors with different formal parameters $b$ are evaluated and shown in Figure 19. The best formal parameters $b$ of surrogate models are 4, 4, 3 and 6 for predicting heave, surge, transverse metacentric 
Table 6: Full factorial design for HVS semi-submersible.

\begin{tabular}{cccccc}
\hline Factors & Lower bound & Initial & Upper bound & Level number & Level step \\
\hline Draft & $27.9 \mathrm{~m}$ & $31 \mathrm{~m}$ & $34.1 \mathrm{~m}$ & 3 & $3.1 \mathrm{~m}$ \\
Column spacing & $65.7 \mathrm{~m}$ & $73 \mathrm{~m}$ & $80.3 \mathrm{~m}$ & 3 & $7.3 \mathrm{~m}$ \\
Pontoon height & $10.8 \mathrm{~m}$ & $12 \mathrm{~m}$ & $13.2 \mathrm{~m}$ & 3 & $1.2 \mathrm{~m}$ \\
Cakepiece width & $6.4 \mathrm{~m}$ & $8 \mathrm{~m}$ & $9.6 \mathrm{~m}$ & 3 & $1.6 \mathrm{~m}$ \\
Column corner radius & $2.8 \mathrm{~m}$ & $4 \mathrm{~m}$ & $5.2 \mathrm{~m}$ & 3 & $1.2 \mathrm{~m}$ \\
Blister width & $5.25 \mathrm{~m}$ & $7.5 \mathrm{~m}$ & $9.75 \mathrm{~m}$ & 3 & $2.25 \mathrm{~m}$ \\
\hline
\end{tabular}
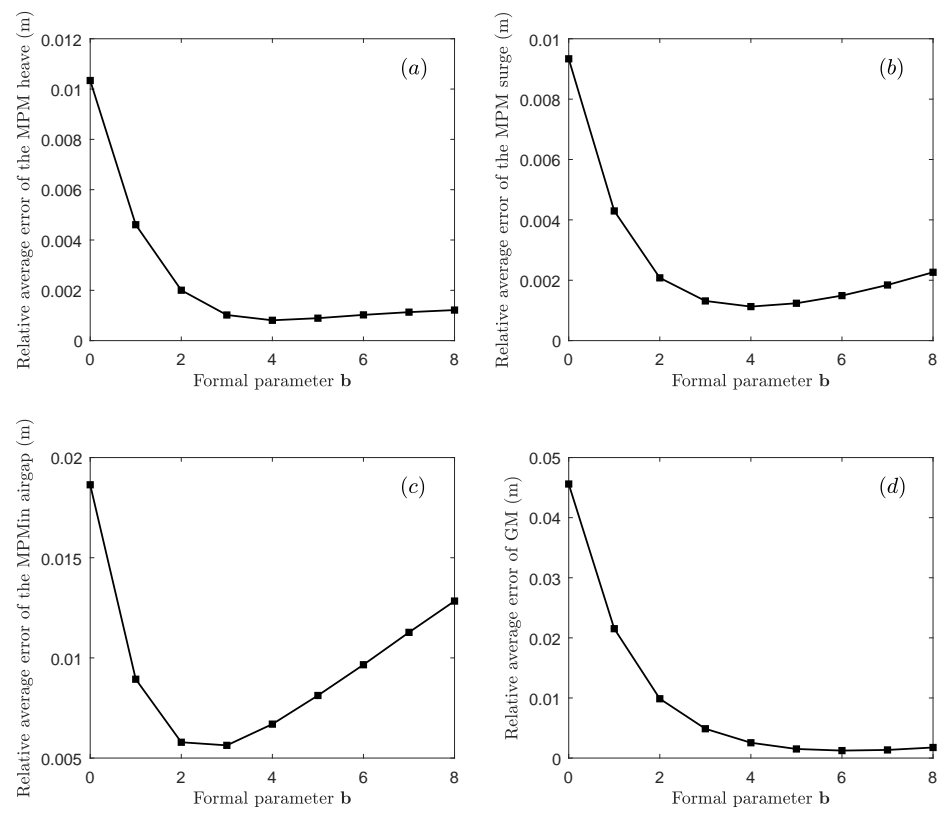

Figure 19: The average errors of RBF predictions by LOOCV with respect to the formal parameter for HVS semi-submersible. (a) The MPM heave motion; (b) The MPM surge motion; (c) The MPMin airgap; $(d)$ Transverse metacentric height. JONSWAP spectrum with $H_{s}=15.8 \mathrm{~m} ; T_{p}=15.4 \mathrm{~s}$; peak enhancement factor $\gamma=2.4$.

height and airgap, respectively. The correlations between the single design variable of hull forms and the MPM heave motion response are illustrated in Figure 20. It can be observed when the draft or blister width increases, the MPM heave motion will decrease. However, when either the width of cakepiece, column corner radius or pontoon height decreases, the MPM heave motion reduces. The variation tendencies of the objectives with respect to the design variables near the Pareto-optimal solutions are also computed. It can be found that the variation tendencies of Pareto-optimal solutions agree well with the variation tendencies of initial design.

\subsubsection{Pareto-optimal solutions}

The Pareto-optimal solutions are ranked with the increase of heave motion, as listed in Table 7. The MPM heave motion and total weight can be reduced by $11.92 \%$ and $13.00 \%$ at most, respectively. The results of the optimization are shown in Figure 21. As presented, 28 Pareto-optimal solutions are found to be better than the initial design in terms of both objectives. Only 2 Pareto-optimal solutions are worse than the initial 

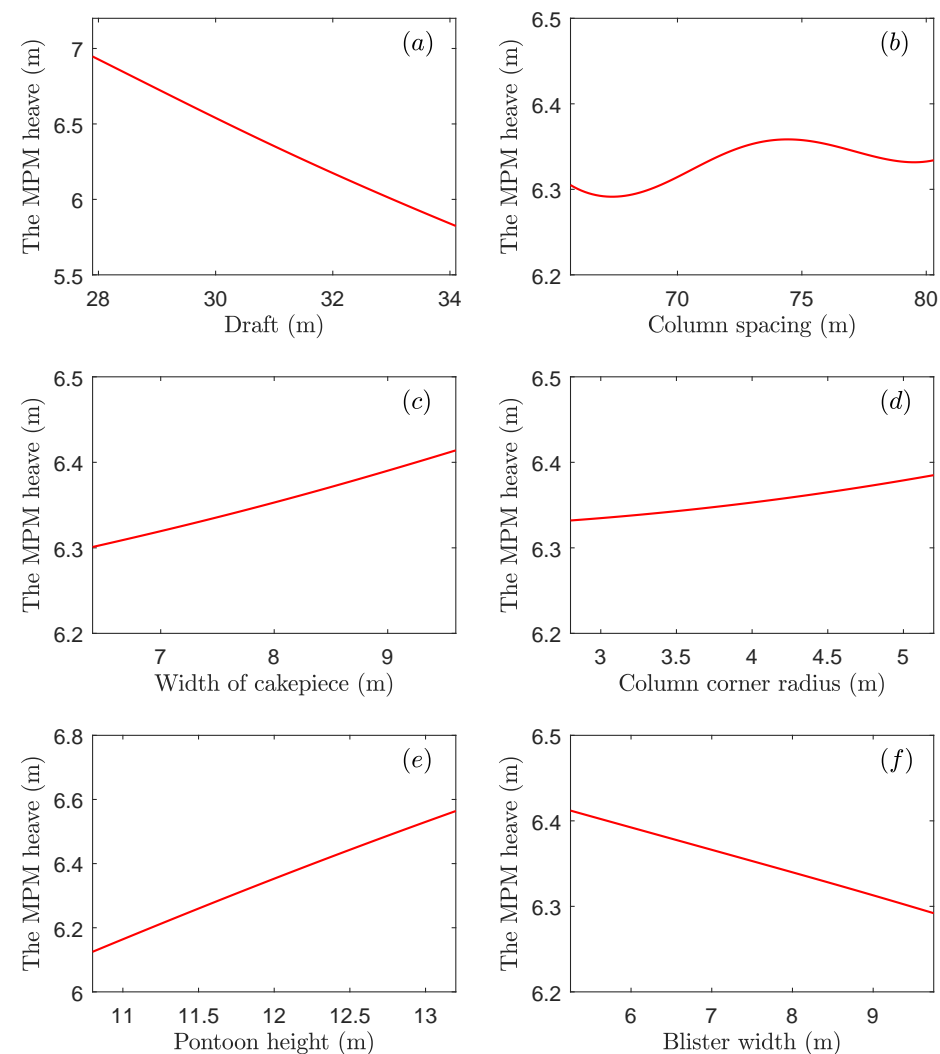

Figure 20: The correlations between the single design variable and the MPM heave motion response. (a) Draft; $(b)$ Column spacing; (c) Width of cakepiece; $(d)$ Column corner radius; $(e)$ Pontoon height; $(f)$ Blister width.

design in MPM heave motion. The MPM heave motions of these hull forms are increased by $0.20 \%$ and $1.07 \%$, respectively, but their total weights are greatly reduced by $12.59 \%$ and $13.00 \%$, respectively.

By comparing the results of surrogate models with the direct numerical simulations, it is found that the maximum relative error is less than $0.5 \%$, which further confirms the accuracy of the surrogate models. Moreover, the MPM surge motion, the MPMin airgap, and the transverse metacentric height are all computed for the 30 Pareto-optimal solutions, which fulfill the constraints.

According to the 30 Pareto-optimal solutions obtained by optimization, the variation tendencies of six design variables are illustrated in Figure 22. 30 Pareto-optimal solutions are sorted in descending order of the total weight. As shown in Figures $22(a)$ and $(f)$, the drafts of the first 8 Pareto-optimal solutions are around 34 $\mathrm{m}$, while the drafts of the latter 22 Pareto-optimal solutions are gradually reduced. However, it can be observed that the blister widths of the first 8 Pareto-optimal solutions are gradually reduced, while the blister widths of the latter 22 Pareto-optimal solutions are kept around $5.35 \mathrm{~m}$. The effects of draft and blister width on MPM heave motion are consistent with the previous analyses shown in Figures $20(a)$ and $(f)$, which confirms that increasing draft or blister width can help reduce the heave motion but increase the total weight. As shown in Figures $20(b)$ and $(c)$, the column spacings of first 14 Pareto-optimal solutions are gradually reduced, while the column spacings of the latter 16 Pareto-optimal solutions are remained around $71.8 \mathrm{~m}$. The pontoon height 
Table 7: The Pareto-optimal solutions for HVS semi-submersible obtained by utilizing MOPSO.

\begin{tabular}{|c|c|c|c|c|c|c|c|c|c|c|}
\hline & Draft & CS & $\mathrm{PH}$ & $\mathrm{CP}$ & CCR & BW & Heave $_{\text {MPM }}$ & Percentage & Weight & Percentage \\
\hline Unit & $\mathrm{m}$ & $\mathrm{m}$ & $\mathrm{m}$ & $\mathrm{m}$ & $\mathrm{m}$ & $\mathrm{m}$ & $\mathrm{m}$ & $\%$ & $10^{4}$ ton & $\%$ \\
\hline Initial & 31.000 & 73.000 & 12.000 & 8.000 & 4.000 & 7.500 & 6.353 & 0.000 & 11.954 & 0.000 \\
\hline 1 & 34.079 & 76.099 & 10.833 & 7.020 & 3.449 & 7.111 & 5.596 & -11.918 & 11.948 & -0.054 \\
\hline 2 & 34.079 & 75.439 & 10.832 & 7.020 & 3.505 & 6.947 & 5.600 & -11.848 & 11.851 & -0.864 \\
\hline 3 & 34.079 & 75.614 & 10.833 & 7.015 & 3.479 & 6.731 & 5.603 & -11.812 & 11.822 & -1.104 \\
\hline 4 & 34.078 & 75.429 & 10.832 & 7.009 & 3.573 & 6.522 & 5.607 & -11.737 & 11.756 & -1.658 \\
\hline 5 & 34.068 & 75.524 & 10.833 & 7.006 & 3.551 & 6.035 & 5.616 & -11.605 & 11.663 & -2.433 \\
\hline 6 & 34.078 & 74.717 & 10.831 & 6.995 & 3.672 & 5.812 & 5.616 & -11.599 & 11.539 & -3.472 \\
\hline 7 & 34.072 & 74.645 & 10.838 & 6.982 & 3.692 & 5.658 & 5.620 & -11.532 & 11.503 & -3.774 \\
\hline 8 & 34.069 & 74.035 & 10.826 & 6.974 & 4.023 & 5.428 & 5.623 & -11.488 & 11.375 & -4.841 \\
\hline 9 & 33.890 & 73.778 & 10.817 & 6.984 & 4.008 & 5.379 & 5.646 & -11.122 & 11.311 & -5.382 \\
\hline 10 & 33.797 & 73.574 & 10.822 & 6.984 & 4.291 & 5.381 & 5.667 & -10.798 & 11.264 & -5.772 \\
\hline 11 & 33.681 & 73.324 & 10.821 & 6.984 & 4.189 & 5.418 & 5.677 & -10.642 & 11.237 & -5.999 \\
\hline 12 & 33.570 & 72.921 & 10.814 & 6.984 & 4.303 & 5.401 & 5.688 & -10.467 & 11.170 & -6.562 \\
\hline 13 & 33.377 & 72.360 & 10.814 & 6.966 & 4.305 & 5.353 & 5.704 & -10.210 & 11.079 & -7.318 \\
\hline 14 & 33.196 & 71.841 & 10.814 & 6.965 & 4.275 & 5.353 & 5.717 & -10.015 & 11.007 & -7.926 \\
\hline 15 & 32.922 & 71.769 & 10.814 & 6.975 & 4.262 & 5.356 & 5.761 & -9.322 & 10.959 & -8.327 \\
\hline 16 & 32.703 & 71.917 & 10.813 & 6.964 & 4.244 & 5.348 & 5.803 & -8.661 & 10.936 & -8.513 \\
\hline 17 & 32.404 & 71.891 & 10.813 & 6.965 & 4.263 & 5.344 & 5.855 & -7.836 & 10.885 & -8.944 \\
\hline 18 & 31.872 & 71.775 & 10.814 & 6.962 & 4.268 & 5.354 & 5.947 & -6.385 & 10.793 & -9.716 \\
\hline 19 & 31.663 & 71.796 & 10.814 & 6.961 & 4.270 & 5.353 & 5.986 & -5.772 & 10.761 & -9.977 \\
\hline 20 & 31.426 & 71.830 & 10.812 & 6.943 & 4.273 & 5.344 & 6.031 & -5.075 & 10.723 & -10.294 \\
\hline 21 & 31.242 & 71.788 & 10.813 & 6.951 & 4.275 & 5.347 & 6.064 & -4.543 & 10.692 & -10.553 \\
\hline 22 & 30.964 & 71.887 & 10.814 & 6.951 & 4.264 & 5.343 & 6.119 & -3.676 & 10.657 & -10.848 \\
\hline 23 & 30.687 & 71.902 & 10.814 & 6.951 & 4.294 & 5.345 & 6.174 & -2.824 & 10.614 & -11.212 \\
\hline 24 & 30.627 & 71.831 & 10.812 & 6.952 & 4.296 & 5.351 & 6.183 & -2.680 & 10.598 & -11.342 \\
\hline 25 & 30.466 & 71.814 & 10.813 & 6.957 & 4.281 & 5.345 & 6.214 & -2.195 & 10.572 & -11.564 \\
\hline 26 & 30.249 & 72.066 & 10.814 & 6.968 & 4.303 & 5.355 & 6.263 & -1.420 & 10.561 & -11.651 \\
\hline 27 & 29.967 & 71.824 & 10.813 & 6.946 & 4.280 & 5.346 & 6.310 & -0.675 & 10.494 & -12.216 \\
\hline 28 & 29.796 & 71.973 & 10.814 & 6.946 & 4.304 & 5.337 & 6.348 & -0.074 & 10.478 & -12.348 \\
\hline 29 & 29.686 & 71.837 & 10.813 & 6.945 & 4.274 & 5.341 & 6.365 & 0.196 & 10.450 & -12.583 \\
\hline 30 & 29.404 & 71.808 & 10.812 & 6.941 & 4.296 & 5.336 & 6.421 & 1.067 & 10.400 & -12.999 \\
\hline
\end{tabular}

values are around the lower bound among 30 Pareto-optimal solutions. This suggests decreasing pontoon height can not only reduce the MPM heave motion but also lead to reduction of total structural weight. This is consistent with the solutions by surrogate models shown in Figure $20(e)$. The cakepiece widths and column corner radii of 30 Pareto-optimal solutions are illustrated in Figures $22(d)$ and $(e)$, respectively. The values of cakepiece width are about $6.95 \mathrm{~m}$. The column corner radii of the first 13 Pareto-optimal solutions are gradually increased, while the latter 17 Pareto-optimal solutions remain around $4.27 \mathrm{~m}$.

Based on the multi-objective optimization for the HVS semi-submersible, it is found that when the draft or blister width increases, the MPM heave motion decreases. However, as the width of cakepiece, column corner radius or pontoon height decreases, the MPM heave motion will reduce. From the 30 Pareto-optimal solutions, it can be observed that the MPM heave motion and total weight can be reduced by $11.92 \%$ and $13.00 \%$ at 


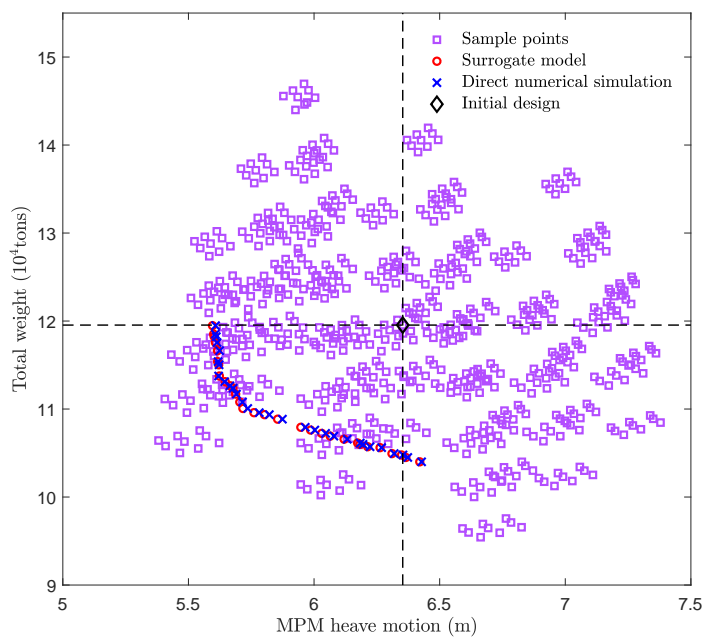

Figure 21: 30 Pareto-optimal solutions for HVS semi-submersible obtained by MOPSO based on RBF model and compared with direct numerical simulations.
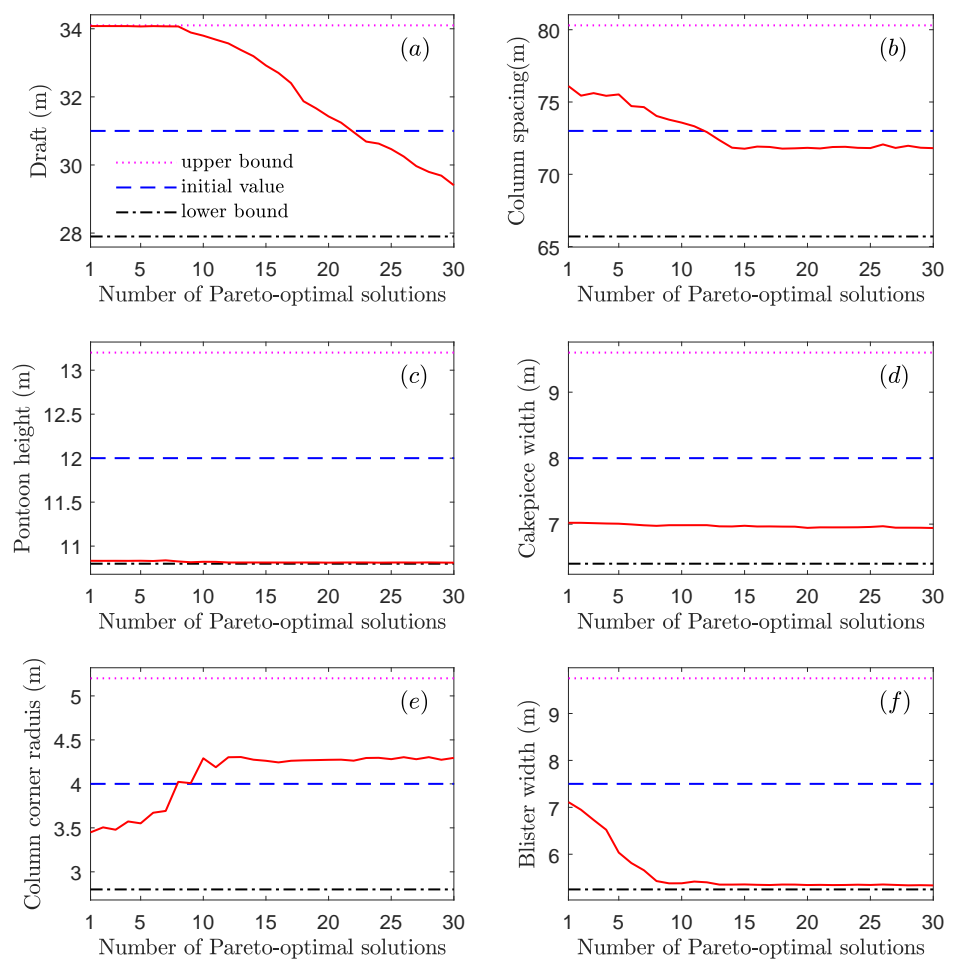

Figure 22: The variation of six design variables with respect to 30 Pareto-optimal solutions. (a) Draft; (b) Column spacing; (c) Pontoon height; $(d)$ Width of cakepiece; $(e)$ Column corner radius; $(f)$ Blister width. 
most, respectively. Increasing draft should be considered as a priority, and then increasing the blister width and column spacing. In addition, the lower bound value of pontoon height should be retained for lower total weight. This is the most efficient design strategy for reducing the MPM heave motion.

\subsection{Semi-submersible floating drilling unit.}

The sketch of SEMI FDU is illustrated in Figure 23. The definition of the incident wave heading is

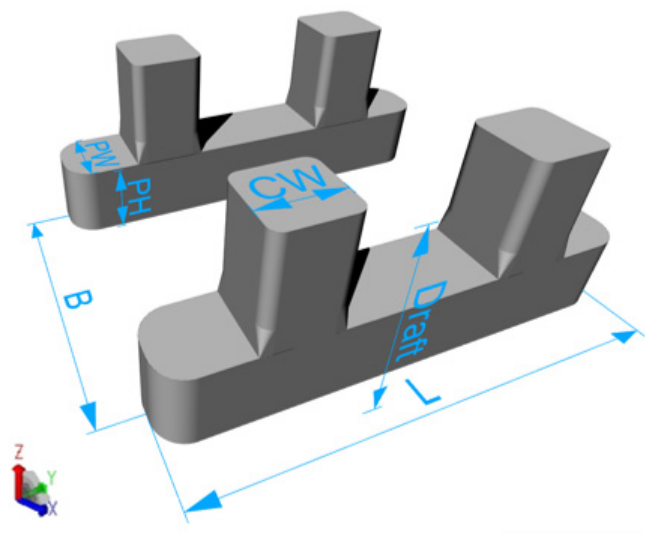

Figure 23: Definition of the main particulars of the base SEMI FDU. CW: column width; PW: pontoon width; PH: pontoon height; L: length overall; B: breadth overall.

illustrated in Figure 24. The main particulars of the initial design are listed in Table 8. The contour of airgaps

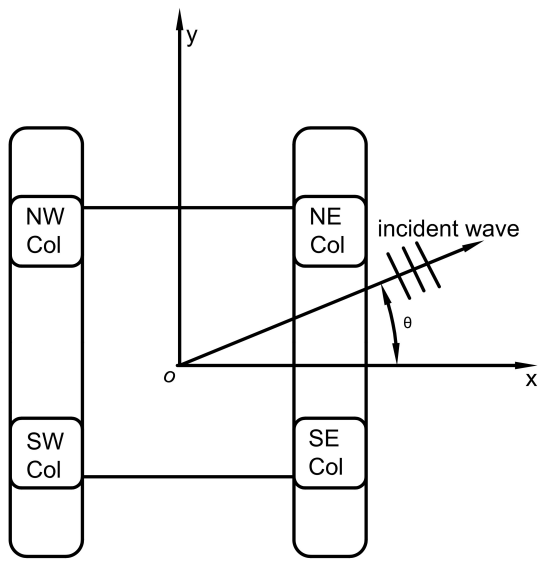

Figure 24: Definition of incident wave heading $\theta$ (plan view) and index of four columns. NW Col: northwest column; NE Col: northeast column; SW Col: southwest column; SE Col: southeast column.

for SEMI FDU in long-crested irregular waves is shown in Figure 25. As shown, the MPMin airgap is $2.69 \mathrm{~m}$ and occurs at the point $(29.4 \mathrm{~m}, 28.3 \mathrm{~m}, 0 \mathrm{~m})$, which is located at the down side of NE column in wave heading $\theta=90^{\circ}$.

\subsubsection{Surrogate model}

Totally $3^{6}=729$ sample points are selected for the surrogate models. The upper and lower bounds of different factors, and level steps of all factors are listed in Table 9. The LOOCV is adopted to find the best 
Table 8: Main particulars of the initial SEMI FDU

\begin{tabular}{lccc}
\hline Description & Unit & Symbol & Value \\
\hline Length overall & $\mathrm{m}$ & LOP & 114.4 \\
Breadth overall & $\mathrm{m}$ & $\mathrm{B}$ & 97 \\
Column length & $\mathrm{m}$ & $\mathrm{CL}$ & 18.2 \\
Column width & $\mathrm{m}$ & $\mathrm{CW}$ & 16.25 \\
Column corner radius & $\mathrm{m}$ & $\mathrm{CCR}$ & 5.045 \\
Column spacing & $\mathrm{m}$ & $\mathrm{CS}$ & 59.8 \\
Pontoon width & $\mathrm{m}$ & $\mathrm{PW}$ & 16.25 \\
Pontoon height & $\mathrm{m}$ & $\mathrm{PH}$ & 10.4 \\
Freeboard & $\mathrm{m}$ & $\mathrm{FB}$ & 20 \\
Draft & $\mathrm{m}$ & $\mathrm{Draft}$ & 23.15 \\
Displacement & $\mathrm{metric}$ & $\Delta$ & 53,400 \\
Transverse metacentric height & $\mathrm{m}$ & $G M_{T}$ & 3 \\
Longitudinal metacentric height & $\mathrm{m}$ & $G M_{L}$ & 8 \\
\hline
\end{tabular}
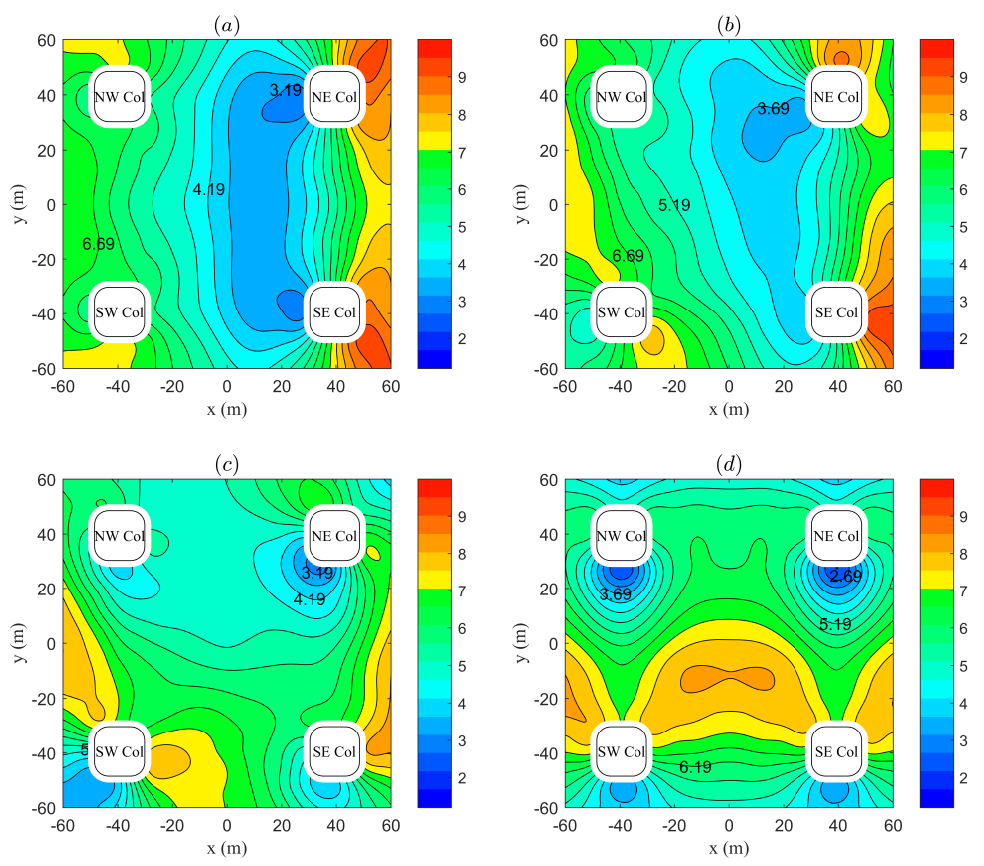

Figure 25: Contour of airgaps for SEMI FDU in long-crested irregular waves, $H_{s}=15.8 \mathrm{~m} ; T_{p}=15.4$ sec; peak enhancement factor $\gamma=2.4$. (a) $\theta=0^{\circ}$; (b) $\theta=22.5^{\circ}$; (c) $\theta=45^{\circ}$; (d) $\theta=90^{\circ}$.

formal parameters $b$ to improve the accuracy of the surrogate models.

As shown in Figure 26, the surrogate models with specific parameters are validated by comparing with the direct numerical simulations. As can be observed in Figure 27, when the draft of platform increases, the MPM heave motion reduces. In addition, as the pontoon width or column width decreases, the MPM heave motion will reduce. As the pontoon height increases, the MPM heave motion slightly increases. Moreover, both the 
Table 9: Full factorial design for SEMI FDU.

\begin{tabular}{cccccc}
\hline Factors & Lower bound & Initial & Upper bound & Level number & Level step \\
\hline Draft & $20.835 \mathrm{~m}$ & $23.15 \mathrm{~m}$ & $25.465 \mathrm{~m}$ & 3 & $2.315 \mathrm{~m}$ \\
Column width & $16.38 \mathrm{~m}$ & $18.2 \mathrm{~m}$ & $20.02 \mathrm{~m}$ & 3 & $1.82 \mathrm{~m}$ \\
Pontoon height & $9.36 \mathrm{~m}$ & $10.4 \mathrm{~m}$ & $11.44 \mathrm{~m}$ & 3 & $1.04 \mathrm{~m}$ \\
Length & $102.96 \mathrm{~m}$ & $114.4 \mathrm{~m}$ & $125.84 \mathrm{~m}$ & 3 & $11.44 \mathrm{~m}$ \\
Breadth & $87.3 \mathrm{~m}$ & $97 \mathrm{~m}$ & $106.7 \mathrm{~m}$ & 3 & $9.7 \mathrm{~m}$ \\
Pontoon width & $14.625 \mathrm{~m}$ & $16.25 \mathrm{~m}$ & $17.875 \mathrm{~m}$ & 3 & $1.625 \mathrm{~m}$ \\
\hline
\end{tabular}

length and breadth of platform have optimal values. When the length and breadth of platform reaches $121 \mathrm{~m}$ and $104 \mathrm{~m}$, respectively, the minimum MPM heave motion can be achieved.
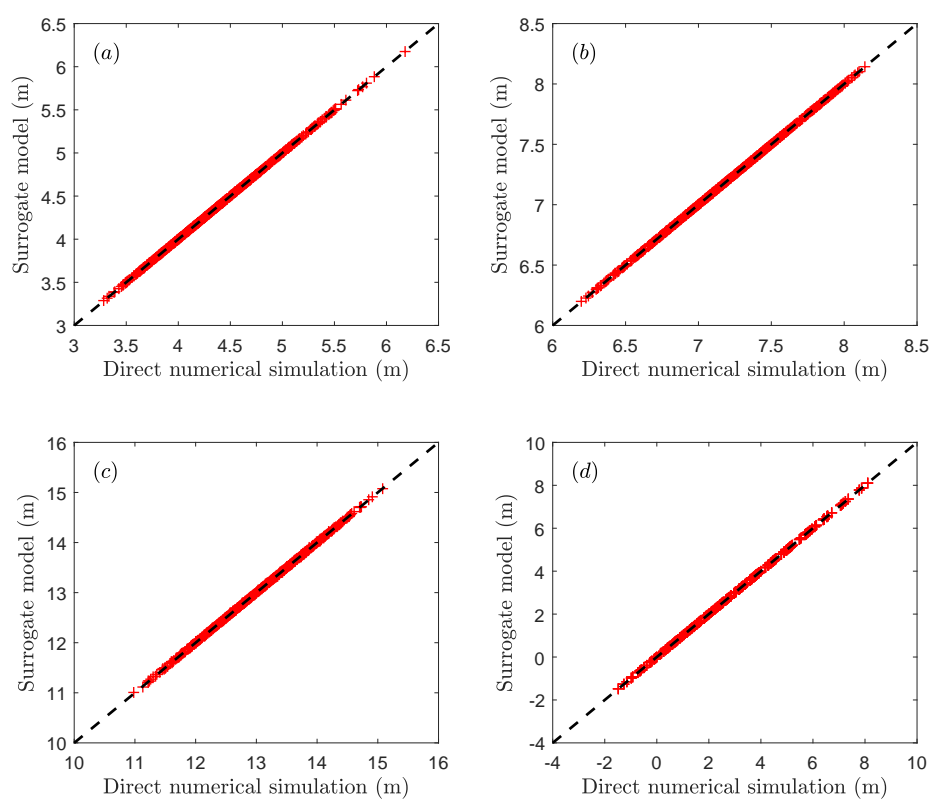

Figure 26: Comparison of the surrogate models with specific parameters and the direct numerical simulations. (a) The MPM heave motion $(b=5) ;(b)$ The MPM surge motion $(b=4) ;(c)$ The MPMin airgap $(b=3) ;(d)$ Transverse metacentric height $(b=8)$.

\subsubsection{Pareto-optimal solutions}

As listed in Table 10, the Pareto-optimal solutions are ranked with the increase of heave motion. It can be observed that the MPM heave motion and total weight can be reduced by $14.96 \%$ and $24.91 \%$ at most, respectively.

The results of the optimization are shown in Figure 28. As shown, 29 Pareto-optimal solutions are better than the initial design in terms of both objectives. Only 1 Pareto-optimal solution is worse than the initial design in MPM heave motion. As shown, the MPM heave motion of this hull form is increased by $0.05 \%$, but its total weight is greatly reduced by $24.91 \%$.

By comparing the results of surrogate models with the direct numerical simulations, it is found that the 

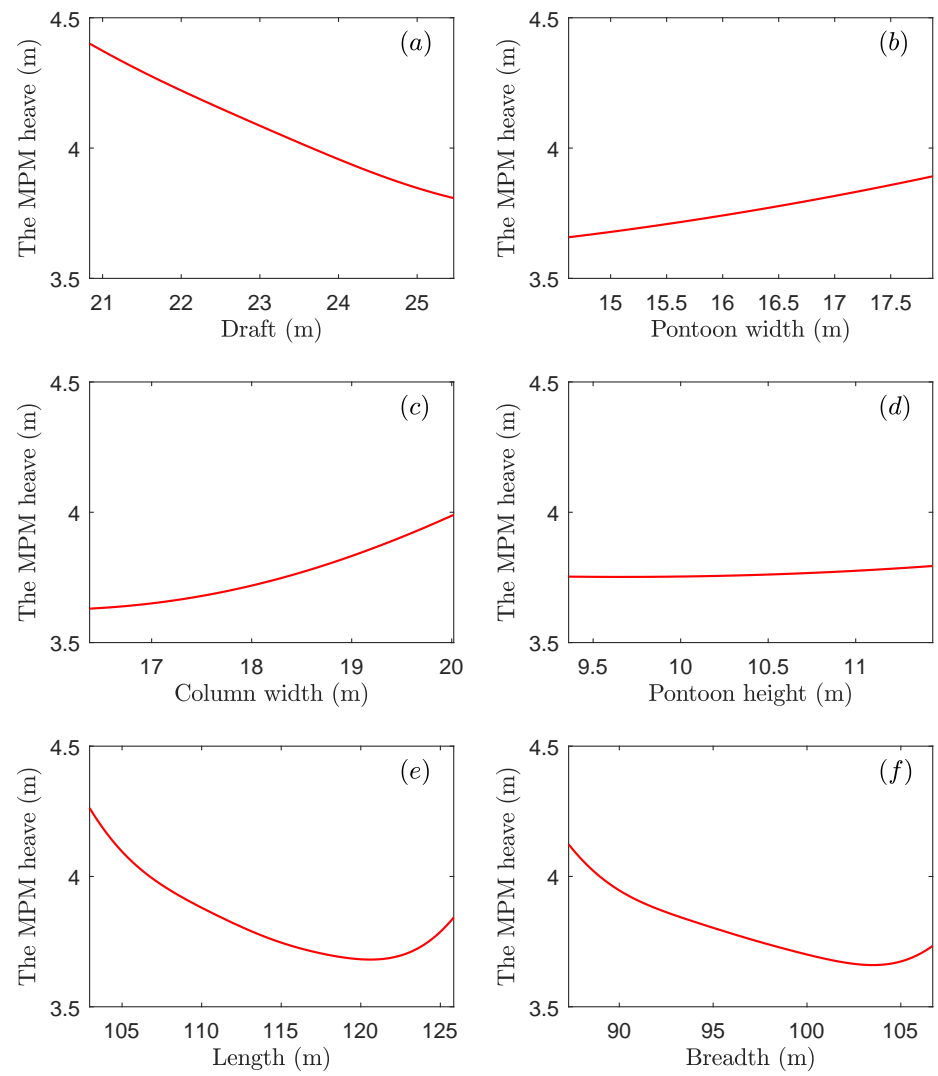

Figure 27: The correlations between the single design variable and the MPM heave motion response. ( $a$ ) Draft; $(b)$ Pontoon width; (c) Column width; $(d)$ Pontoon height; (e) Length overall; $(f)$ Breadth overall.

maximum relative error is less than $1 \%$, which further confirms the accuracy of the surrogate models. Moreover, the MPM surge motion, the MPMin airgap, and the transverse metacentric height are all computed for the 30 Pareto-optimal solutions, which fulfill the constraints.

As shown in Figure $29(a)$, the drafts of 30 Pareto-optimal solutions gradually reduce. The effect of draft on MPM heave motion is consistent with the previous analyses illustrated in Figure $27(a)$, which indicates that increasing draft can help reduce the heave motion, but the total weight will increase. As presented in Figures $29(b)$ and $(d)$, the pontoon heights and widths of the first 15 Pareto-optimal solutions are gradually reduced, while the pontoon heights and widths of the latter 15 Pareto-optimal solutions are the lower bound value. This means that properly decreasing the pontoon size is beneficial to the MPM heave motion and total weight of the platform. As shown in Figure $29(c)$, as the sequence number of Pareto-optimal solutions increases, the column width slightly increases from $17.4 \mathrm{~m}$ to $17.9 \mathrm{~m}$. This suggests increasing column width can not only increase the MPM heave motion but also increase the total weight, which is consistent with the predictions by surrogate models, as illustrated in Figure $27(c)$. Figures $29(d)$ and $(e)$ show that the values of length overall are approximate to the lower bound value, and the values of breadth overall are approximate to the upper bound value. In order to validate the optimization results, heave RAOs of the initial design and case 


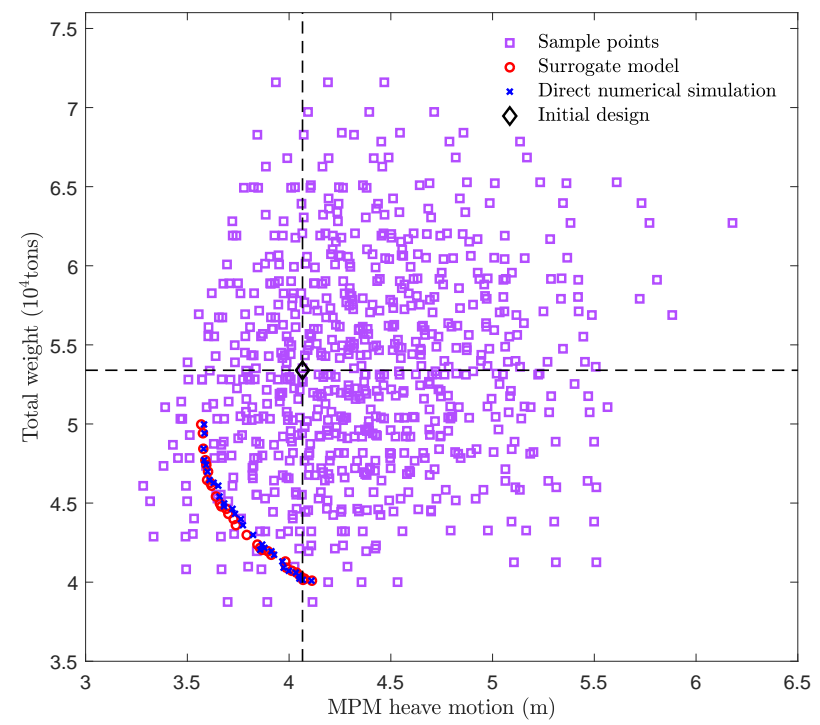

Figure 28: 30 Pareto-optimal solutions for SEMI FDU obtained by MOPSO based on RBF model and compared with direct numerical simulations.
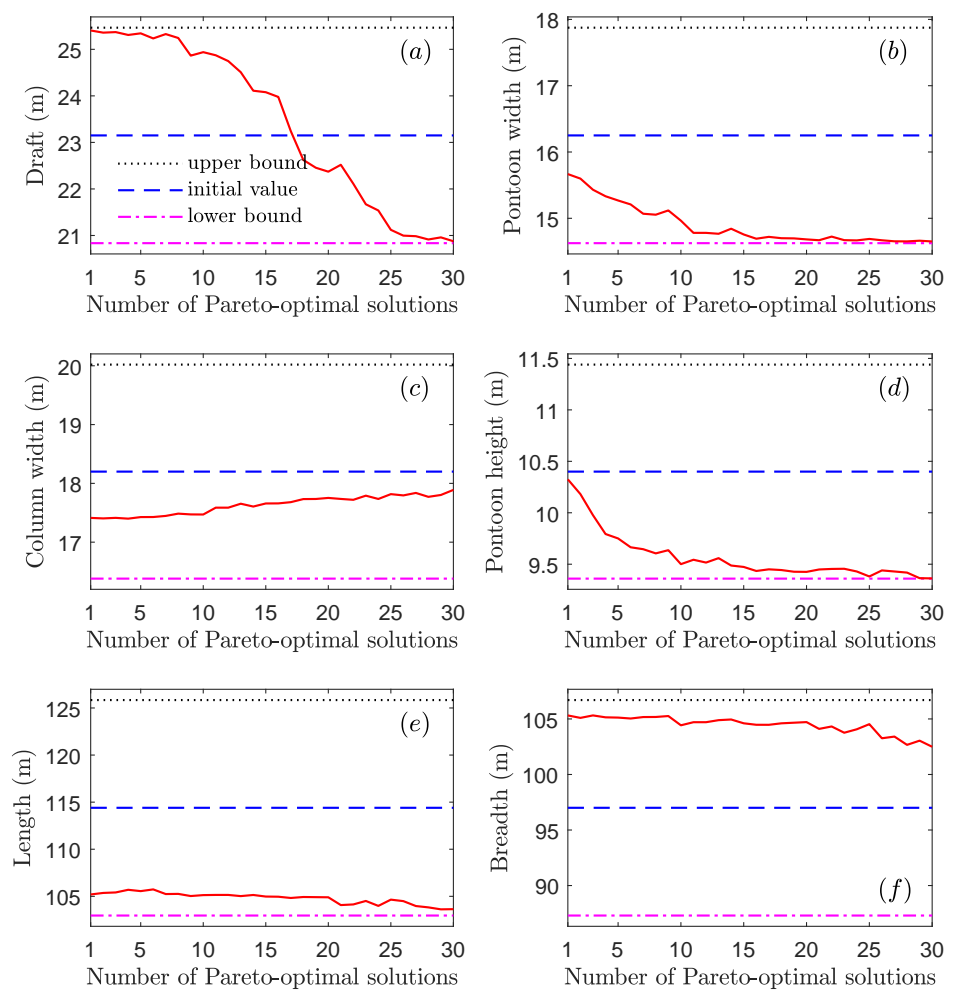

Figure 29: The variation of six design variables with respect to 30 Pareto-optimal solutions. (a) Draft; (b) Pontoon width; (c) Column width; $(d)$ Pontoon height; $(e)$ Length; $(f)$ Breadth. 
Table 10: The Pareto-optimal solutions for SEMI FDU obtained by utilizing MOPSO.

\begin{tabular}{|c|c|c|c|c|c|c|c|c|c|c|}
\hline & Draft & $\mathrm{CW}$ & $\mathrm{PH}$ & $\mathrm{L}$ & B & PW & Heave $_{\mathrm{MPM}}$ & Percentage & Weight & Percentage \\
\hline Unit & $\mathrm{m}$ & $\mathrm{m}$ & $\mathrm{m}$ & $\mathrm{m}$ & $\mathrm{m}$ & $\mathrm{m}$ & $\mathrm{m}$ & $\%$ & $10^{4}$ ton & $\%$ \\
\hline Initial & 23.150 & 18.200 & 10.400 & 114.400 & 97.000 & 16.250 & 4.066 & 0.000 & 5.340 & 0.000 \\
\hline 1 & 25.405 & 17.413 & 10.325 & 105.197 & 105.315 & 15.667 & 3.458 & -14.963 & 4.997 & -6.416 \\
\hline 2 & 25.358 & 17.404 & 10.181 & 105.360 & 105.096 & 15.599 & 3.459 & -14.928 & 4.941 & -7.459 \\
\hline 3 & 25.370 & 17.413 & 9.977 & 105.412 & 105.317 & 15.431 & 3.463 & -14.826 & 4.843 & -9.294 \\
\hline 4 & 25.309 & 17.400 & 9.793 & 105.693 & 105.145 & 15.333 & 3.466 & -14.753 & 4.771 & -10.649 \\
\hline 5 & 25.344 & 17.427 & 9.750 & 105.553 & 105.122 & 15.269 & 3.475 & -14.544 & 4.742 & -11.184 \\
\hline 6 & 25.234 & 17.428 & 9.665 & 105.735 & 105.039 & 15.209 & 3.482 & -14.370 & 4.699 & -12.004 \\
\hline 7 & 25.328 & 17.447 & 9.646 & 105.242 & 105.170 & 15.069 & 3.488 & -14.218 & 4.646 & -12.994 \\
\hline 8 & 25.244 & 17.485 & 9.605 & 105.259 & 105.178 & 15.054 & 3.509 & -13.707 & 4.628 & -13.324 \\
\hline 9 & 24.865 & 17.472 & 9.636 & 105.031 & 105.262 & 15.119 & 3.532 & -13.143 & 4.610 & -13.666 \\
\hline 10 & 24.939 & 17.470 & 9.501 & 105.129 & 104.428 & 14.964 & 3.534 & -13.087 & 4.541 & -14.952 \\
\hline 11 & 24.872 & 17.587 & 9.543 & 105.148 & 104.707 & 14.780 & 3.560 & -12.436 & 4.497 & -15.774 \\
\hline 12 & 24.747 & 17.587 & 9.516 & 105.148 & 104.707 & 14.780 & 3.570 & -12.194 & 4.479 & -16.113 \\
\hline 13 & 24.508 & 17.654 & 9.559 & 105.024 & 104.891 & 14.767 & 3.605 & -11.337 & 4.463 & -16.410 \\
\hline 14 & 24.111 & 17.606 & 9.487 & 105.143 & 104.950 & 14.843 & 3.618 & -11.030 & 4.432 & -17.001 \\
\hline 15 & 24.079 & 17.658 & 9.473 & 104.976 & 104.608 & 14.756 & 3.644 & -10.390 & 4.399 & -17.623 \\
\hline 16 & 23.977 & 17.659 & 9.434 & 104.959 & 104.475 & 14.692 & 3.653 & -10.160 & 4.360 & -18.343 \\
\hline 17 & 23.242 & 17.680 & 9.450 & 104.825 & 104.472 & 14.720 & 3.706 & -8.866 & 4.298 & -19.499 \\
\hline 18 & 22.624 & 17.733 & 9.443 & 104.928 & 104.606 & 14.700 & 3.753 & -7.707 & 4.237 & -20.640 \\
\hline 19 & 22.456 & 17.735 & 9.427 & 104.912 & 104.661 & 14.697 & 3.764 & -7.416 & 4.216 & -21.034 \\
\hline 20 & 22.371 & 17.753 & 9.426 & 104.898 & 104.716 & 14.682 & 3.775 & -7.164 & 4.204 & -21.261 \\
\hline 21 & 22.519 & 17.735 & 9.449 & 104.072 & 104.105 & 14.669 & 3.806 & -6.384 & 4.195 & -21.432 \\
\hline 22 & 22.111 & 17.720 & 9.454 & 104.139 & 104.323 & 14.724 & 3.818 & -6.090 & 4.173 & -21.842 \\
\hline 23 & 21.671 & 17.791 & 9.456 & 104.509 & 103.758 & 14.670 & 3.866 & -4.916 & 4.131 & -22.642 \\
\hline 24 & 21.537 & 17.735 & 9.429 & 103.983 & 104.070 & 14.667 & 3.875 & -4.695 & 4.092 & -23.367 \\
\hline 25 & 21.121 & 17.817 & 9.381 & 104.649 & 104.531 & 14.689 & 3.898 & -4.137 & 4.072 & -23.741 \\
\hline 26 & 20.997 & 17.795 & 9.440 & 104.490 & 103.260 & 14.669 & 3.942 & -3.040 & 4.060 & -23.957 \\
\hline 27 & 20.985 & 17.837 & 9.429 & 103.969 & 103.412 & 14.653 & 3.976 & -2.210 & 4.041 & -24.324 \\
\hline 28 & 20.914 & 17.771 & 9.419 & 103.827 & 102.666 & 14.652 & 4.001 & -1.600 & 4.022 & -24.666 \\
\hline 29 & 20.959 & 17.803 & 9.364 & 103.618 & 103.045 & 14.662 & 4.011 & -1.350 & 4.015 & -24.809 \\
\hline 30 & 20.877 & 17.889 & 9.362 & 103.636 & 102.494 & 14.651 & 4.068 & 0.053 & 4.010 & -24.909 \\
\hline
\end{tabular}

No.11 Pareto-optimal solutions are compared and illustrated in Figure 30. As shown in Figure 30, the CFD simulations for the initial and optimal designs have been carried out at the same incoming wave periods. The results obtained from panel method and Morison's equation are in the same trend with those obtained by using CFD tool for both initial and Case No.11 optimal designs, so that the panel method and Morison's equation can be used for building the surrogate model.

Based on the multi-objective optimization for the SEMI FDU, it is found that when the draft of column increases, the MPM heave motion reduces. However, as the pontoon width or column width decreases, the MPM heave motion will reduce. As the pontoon height increases, the MPM heave motion slightly increases. In addition, the length and breadth of platform have optimal values. When the length of platform is 121 $\mathrm{m}$, the minimum MPM heave motion can be reached. Similarly, when the breadth of platform is $104 \mathrm{~m}$, the 


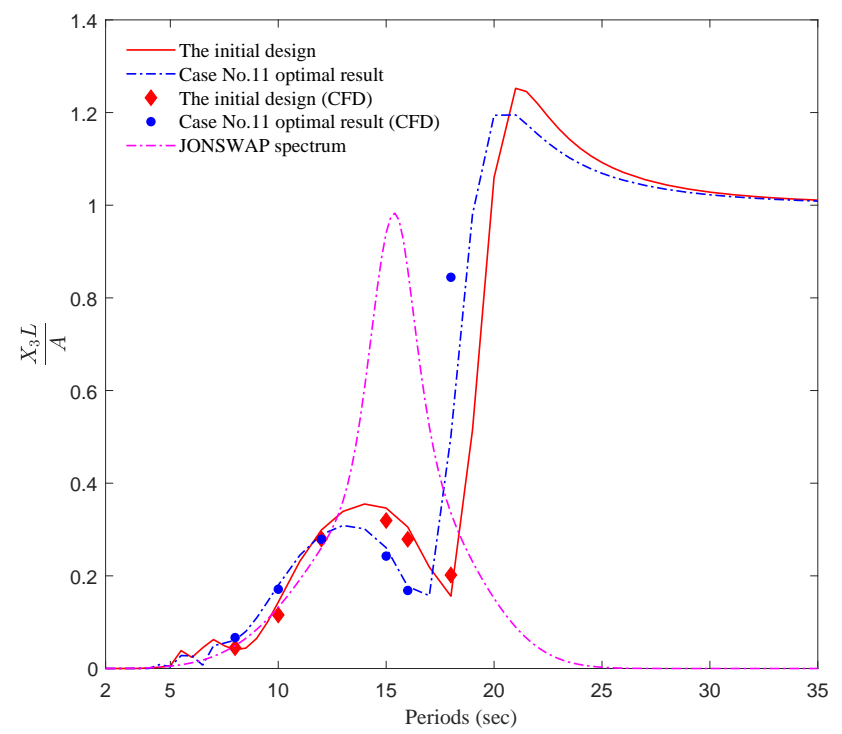

Figure 30: Heave motion RAOs of one optimal design and the initial SEMI FDU design. JONSWAP spectrum $H_{s}=15.8 \mathrm{~m} ; T_{p}$ $=15.4 \mathrm{sec}$; peak enhancement factor $\gamma=2.4 . A$ is the amplitude of incident wave. $L$ is the characteristic length of the SEMI.

minimum MPM heave motion can be achieved. From the 30 Pareto-optimal solutions, it shows that the MPM heave motion and total weight can be reduced by $14.96 \%$ and $24.91 \%$ at most, respectively. In order to obtain the optimized hull sizing, increasing draft should be considered as a priority to ensure safety, and properly decreasing the pontoon size is beneficial to the MPM heave motion and total weight of the platform.

\section{Conclusions}

In the present study, three types of semi-submersible platforms are analyzed and optimized. In order to improve the computing efficiency, the hydrodynamic performances for different hull forms during optimization process are estimated by the surrogate models, which are built by artificial neural network prediction method and IMQ radial basis function. In addition, MOPSO is employed to search for the Pareto-optimal solutions. The variations of design variables with respect to Pareto-optimal solutions for SEMI have been illustrated. The heave RAOs obtained from panel method and Morison's equation are in the same trend with those obtained by using CFD tool for both initial and Case No.11 optimal designs, so that the panel method and Morison's equation can be used for building the surrogate model. A computational program, called Innovative Semi-submersible platform Optimization Program (ISOP), has been developed to solve the multi-objective optimization problem. The objective functions include the MPM heave motion and the total structural weight of the platform, with three constraints being the transverse metacentric height, the MPM surge motion and the MPMin airgap.

In summary, the correlations between the single design variable and the MPM heave motion response have been identified. Among the design variables, the effect of draft on optimization results is the most significant. Based on the surrogate models and MOPSO, Pareto-optimal solutions can be obtained rapidly and most of the optimized hull forms are better than the initial design in terms of MPM heave motion and structural weight. 


\section{Acknowledgement}

We gratefully acknowledge the financial support from National Natural Science Foundation of China with Grant No. 51679138 and would like to thank the reviewers, who provided constructive and valuable suggestions that help to improve the paper.

\section{Reference}

Akagi, S., Yokoyama, R., Ito, K., 1984. Optimal design of semisubmersible's form based on systems analysis. J. Mechanisms, Transmissions, and Automation in Design 106 (4), 524-530.

Anderson, M. J., Whitcomb, P. J., 1970. Design of experiments. Concise Encyclopedia of Statistics 2 (Sept), 33-53.

API RP 2SK, 2005. Recommended Practice for Design and Analysis of Stationkeeping Systems for Floating Structures. In: Third Edition.

API RP 2T, 1997. Recommended Practice for Planning, Design and Constructoing Tension Leg Platforms. In: Second Edition.

Audet, C., Booker, A. J., Dennis, J. E., Frank, P. D., Moore, D., 2000. A surrogate-model-based method for constrained optimization. American Institute of Aeronautics and Astronautics.

Buhmann, M. D., 2009. Radial basis functions. Acta Numerica 9, 1-38.

Cermelli, C., Roddier, D., Busso, C., et al., 2004. Minifloat: A novel concept of minimal floating platform for marginal field development. In: The Fourteenth International Offshore and Polar Engineering Conference. International Society of Offshore and Polar Engineers.

Clauss, G. F., Birk, L., 1994. Optimization the motion behavior of offshore structures. In: Proceedings of BOSS' 94 Conferenece, Cambridge, MA, USA.

Clauss, G. F., Birk, L., 1996. Hydrodynamic shape optimization of large offshore structures. Applied Ocean Research 18 (4), 157-171.

Eberhart, R., Kennedy, J., 1995. A new optimizer using particle swarm theory. In: International Symposium on MICRO Machine and Human Science. pp. 39-43.

Kim, J. D., Jang, B. S., 2016. Application of multi-objective optimization for TLP considering hull-form and tendon system. Ocean Engineering 116, 142-156.

Kyoung, J., Kim, J. W., Jang, H., Lambrakos, K., OSullivan, J., 2015. Investigation on the VIM mitigation of the HVS semisubmersible. In: ASME 2015 34th International Conference on Ocean, Offshore and Arctic Engineering. American Society of Mechanical Engineers, pp. V001T01A021-V001T01A021.

Lee, J. Y., Koo, B. J., Clauss, G., 2007. Automated design of a tension leg platform with minimized tendon fatigue damage and its verification by a fully coupled analysis. Ship Technology Research 54, 11-27. 
Nishimoto, K., Leite, A. J. P., 1993. Effect of lateral keel and blisters on semisubmersible for the minimization of heave motion. In: Proceedings of the 3rd International Offshore and Polar Engineering Conference, Sinpore, June, 1993.

Park, Y., Jang, B. S., Kim, J. D., 2015. Hull-form optimization of semi-submersible FPU considering seakeeping capability and structural weight. Ocean Engineering 104, 714-724.

Reyes-Sierra, M., Coello Coello, C. A., 2006. Multi-objective particle swarm optimizers: A survey of the stateof-the-art. International Journal of Computational Intelligence Research 2 (3), 287-308.

Santana, R. A., Pontes, M. R., Bastos-Filho, C. J. A., 2009. A multiple objective particle swarm optimization approach using crowding distance and roulette wheel. In: Ninth International Conference on Intelligent Systems Design and Applications. pp. 237-242.

Sugita, T., Suzuki, H., 2016. A study on TLP hull sizing by utilizing optimization algorithm. Journal of Marine Science and Technology 21 (4), 611-623.

Vannucci, P., 1996. Simplified optimal design of a tension leg platform (TLP). Structural Optimization 12 , $265-268$.

Williams, N., 2012. Optimized global sizing approach for gulf of mexico production semisubmersible. In: ASME 2012 31st International Conference on Ocean, Offshore and Arctic Engineering. American Society of Mechanical Engineers, pp. 107-112.

Xu, Q., 2011. A new semisubmersible design for improved heave motion, vortex-induced motion and quayside stability. In: ASME 2011 International Conference on Ocean, Offshore and Arctic Engineering. pp. 95-103.

Yang, X. S., 2010. Engineering Optimization: An Introduction with Metaheuristic Applications. Wiley Publishing.

Zhang, X., Bandyk, P., Beck, R. F., 2010. Time-domain simulations of radiation and diffraction forces. Journal of Ship Research 54 (2), 79-94.

Zhang, X., Song, X., Qiu, W., Yuan, Z., You, Y., Deng, N., 2018. Multi-objective optimization of tension leg platform using evolutionary algorithm based on surrogate model. Ocean Engineering 148, 612-631.

Zhang, X., Song, X., Yuan, Z., You, Y., 2017. Global motion and airgap computations for semi-submersible floating production unit in waves. Ocean Engineering 141, 176-204. 